\title{
WEAK AND CLASSICAL SOLUTIONS OF EQUATIONS OF MOTION FOR THIRD GRADE FLUIDS
}

\author{
JEAN MARIE BERNARD ${ }^{1}$
}

\begin{abstract}
This paper shows that the decomposition method with special basis, introduced by Cioranescu and Ouazar, allows one to prove global existence in time of the weak solution for the third grade fluids, in three dimensions, with small data. Contrary to the special case where $\left|\alpha_{1}+\alpha_{2}\right| \leq$ $(24 \nu \beta)^{1 / 2}$, studied by Amrouche and Cioranescu, the $H^{1}$ norm of the velocity is not bounded for all data. This fact, which led others to think, in contradiction to this paper, that the method of decomposition could not apply to the general case of third grade, complicates substantially the proof of the existence of the solution. We also prove further regularity results by a method similar to that of Cioranescu and Girault for second grade fluids. This extension to the third grade fluids is not straightforward, because of a transport equation which is much more complex.

Résumé. Dans cet article, on montre que la méthode de décomposition avec base spéciale introduite par Cioranescu et Ouazar, permet de démontrer l'existence globale en temps de la solution faible pour les fluides de grade trois, en dimension trois, avec des données petites. Contrairement au cas particulier où $\left|\alpha_{1}+\alpha_{2}\right| \leq(24 \nu \beta)^{1 / 2}$, étudié par Amrouche et Cioranescu, la norme $H^{1}$ de la vitesse n'est pas majorée pour toute donnée. Ce fait, qui conduisait à penser, en contradiction avec cet article, que la méthode de décomposition ne pouvait pas s'appliquer au cas général du grade trois, complique substantiellement la démonstration d'existence de la solution. On établit des résultats de régularité par une méthode similaire à celle de Cioranescu et Girault pour des fluides
\end{abstract}

AMS Subject Classification. 35D05, 35Q99, 76A05.

Received: October 23, 1998.

\section{INTRODUCTION}

The most general constitutive law for the fluids of grade 3 is:

$$
T=-\tilde{p} I+\nu A_{1}+\alpha_{1} A_{2}+\alpha_{2} A_{1}^{2}+\beta_{1} A_{3}+\beta_{2}\left(A_{1} A_{2}+A_{2} A_{1}\right)+\beta\left(\operatorname{tr}\left(A_{1}^{2}\right)\right) A_{1},
$$

(cf. [13]) where $\mathrm{T}$ is the stress tensor, $\tilde{p}$ denotes the pressure (a scalar function), I the unit matrix and $A_{n}$ the $n$th Rivlin-Ericksen tensor given recursively by:

$$
A_{1}=L+L^{T} \text { with } L_{i j}=\frac{\partial u_{i}}{\partial x_{j}}
$$

Keywords and phrases. Galerkin method, special basis, energy estimates.

1 Université d'Evry Val d'Essonne, Boulevard des Coquibus, 91025 Evry Cedex, France. 
where $\mathbf{u}$ is the velocity field and, for $n \geq 2$,

$$
A_{n}=\frac{\mathrm{d} A_{n-1}}{\mathrm{~d} t}+A_{n-1} L+L^{T} A_{n-1} .
$$

The constant $\nu$ is the kinematic viscosity and $\alpha_{i}, \beta_{i}$ and $\beta$ are the normal stress moduli. These constants are not arbitrary. More precisely (cf. [9]) the following result holds: if the Clausius-Duhem inequality is satisfied and the free energy is minimum at equilibrium then

$$
\nu \geq 0, \quad \beta_{1}=\beta_{2}=0, \quad \beta \geq 0, \quad \alpha_{1} \geq 0 .
$$

With these restrictions, we can express $\mathrm{T}$ in the form:

$$
T=-\tilde{p} I+\nu A_{1}+\alpha_{1}\left(\frac{\mathrm{d}}{\mathrm{d} t} A_{1}+A_{1} W-W A_{1}\right)+\left(\alpha_{1}+\alpha_{2}\right) A_{1}^{2}+\beta\left|A_{1}\right|^{2} A_{1},
$$

where

$$
W=\frac{1}{2}\left(L-L^{T}\right)
$$

The incompressibility requires that:

$$
\operatorname{div} \mathbf{u}=0 .
$$

The dynamical equation for a fluid of third grade is of the form:

$$
\frac{\mathrm{d} \mathbf{u}}{\mathrm{d} t}=\operatorname{div} T+\mathbf{f}
$$

We can verify that:

$$
\operatorname{div} A_{1}^{2}=\Delta(\mathbf{u} . \nabla \mathbf{u})-2 \mathbf{u} \cdot \nabla(\Delta \mathbf{u})+\operatorname{curl}(\Delta \mathbf{u}) \times \mathbf{u}+\nabla\left(\mathbf{u} . \Delta \mathbf{u}+\frac{1}{4}\left|A_{1}\right|^{2}\right),
$$

where

$$
\left|A_{1}\right|^{2}=\operatorname{tr}\left(A_{1} A_{1}^{T}\right)
$$

Therefore

$$
\begin{aligned}
\operatorname{div} T= & -\nabla \tilde{p}+\nu \Delta \mathbf{u}+\alpha_{1} \frac{\partial \Delta \mathbf{u}}{\partial t}+\left(\alpha_{1}+\alpha_{2}\right)(\Delta(\mathbf{u} \cdot \nabla \mathbf{u})-2 \mathbf{u} \cdot \nabla(\Delta \mathbf{u})) \\
& +\left(2 \alpha_{1}+\alpha_{2}\right)\left(\operatorname{curl}(\Delta \mathbf{u}) \times \mathbf{u}+\nabla\left(\mathbf{u} . \Delta \mathbf{u}+\frac{1}{4}\left|A_{1}\right|^{2}\right)\right)+\beta \operatorname{div}\left(\left|A_{1}\right|^{2} A_{1}\right) .
\end{aligned}
$$

On using (1.4) and the relation:

$$
\frac{\mathrm{d} \mathbf{u}}{\mathrm{d} t}=\frac{\partial \mathbf{u}}{\partial t}+\operatorname{curl} \mathbf{u} \times \mathbf{u}+\nabla\left(\frac{1}{2}|\mathbf{u}|^{2}\right),
$$

from equation (1.3), we derive

$$
\begin{aligned}
\frac{\partial \mathbf{u}}{\partial t}-\nu \Delta \mathbf{u}-\alpha_{1} \frac{\partial}{\partial t} \Delta \mathbf{u}+\operatorname{curl}\left(\mathbf{u}-\left(2 \alpha_{1}+\alpha_{2}\right) \Delta \mathbf{u}\right) & \times \mathbf{u}-\left(\alpha_{1}+\alpha_{2}\right) \Delta(\mathbf{u} . \nabla \mathbf{u})+2\left(\alpha_{1}+\alpha_{2}\right) \mathbf{u} . \nabla(\Delta \mathbf{u})-\beta \operatorname{div}\left(\left|A_{1}\right|^{2} A_{1}\right) \\
& +\nabla \tilde{p}-\left(2 \alpha_{1}+\alpha_{2}\right) \nabla\left(\mathbf{u} . \Delta \mathbf{u}+\frac{1}{4}\left|A_{1}\right|^{2}\right)+\frac{1}{2} \nabla\left(|\mathbf{u}|^{2}\right)=\mathbf{f} .
\end{aligned}
$$


This equation of motion is completed by the state equation (1.2), some initial data for the velocity and a homogeneous condition for the velocity on the boundary of the domain.

Problem (1.5) is difficult because its nonlinear terms involve third order derivatives. In analogy with the Euler equation, it is convenient to introduce the vorticity $\boldsymbol{\omega}=\mathbf{c u r l} \mathbf{u}$. Taking the curl of equation (1.3), we obtain roughly the following transport equation:

$$
\frac{\partial}{\partial t}\left(\boldsymbol{\omega}-\alpha_{1} \Delta \boldsymbol{\omega}\right)+\frac{\nu}{\alpha_{1}}\left(\boldsymbol{\omega}-\alpha_{1} \Delta \boldsymbol{\omega}\right)+\mathbf{u} \cdot \nabla\left(\boldsymbol{\omega}-\alpha_{1} \Delta \boldsymbol{\omega}\right)+M(\mathbf{u})=\mathbf{c u r l} \mathbf{f}+\frac{\nu}{\alpha_{1}} \mathbf{c u r l} \mathbf{u},
$$

where $M(\mathbf{u})$ collects lower order nonlinear terms that we shall specify in Section 2 .

Fluids of third grade have been studied by Amrouche in [1] and Amrouche and Cioranescu in [3]. They used a special method, which had been used, for the first time, by Cioranescu and Ouazar in [6,7] to solve a problem of second grade fluids. This is a Galerkin's method with the special basis of eigenfunctions corresponding to the scalar product associated with the operator $\operatorname{curl}\left(\mathbf{u}-\alpha_{1} \Delta \mathbf{u}\right)$. This basis allowed them to obtain, from the discrete Galerkin problem, a discrete version of the transport equation (1.6), from which they recovered sharp energy estimates. In three dimensions, they obtained existence and uniqueness of the variational solution during some time interval, without restriction on the data, but under the supplementary condition

$$
\left|\alpha_{1}+\alpha_{2}\right| \leq(24 \nu \beta)^{1 / 2}
$$

On the other hand, they proved global existence in time, under some restrictions on the data, but only in two dimensions.

Recently, several authors such as Galdi et al. in [10], Bresch and Lemoine in [4], Sequeira and Videman in [14] and Videman in [16] used another approach: each one decomposed the original system of equations in their own way but all applied a Schauder fixed point argument. We think that these methods are less efficient than the method of energy estimates of Cioranescu and Ouazar. On the one hand, this method is the only one that gives the existence of solutions in dimension two for the second grade fluids, without restriction on the size of the data. On the other hand, in three dimensions, if the two approaches prove the existence only for sufficiently small data, the method of energy estimates leads to conditions of existence more precise, more explicit and, with no doubt, less restrictive. The reason why this method may be better could be due to the fact that, in those methods using a Schauder fixed point argument, the non-linear terms are placed unsubtly on the right hand side, thus leading to lower-quality results.

The purpose of the present paper is to show that this method of decomposition allows one to prove global existence in time of the weak solution of third grade fluids in three dimensions with small data, but without assuming the condition (1.7) which gives a $H^{1}$ bound of the velocity $\mathbf{u}$ for any data. Here, the $H^{1}$ bound does not follow directly from equation (1.3). Instead, the exponential decay with respect to time of the $H^{1}$ norm of the velocity is obtained by combining (1.3) with the transport equation (1.6), but only with small data. Next, by a method similar to that of Cioranescu and Girault in [5] for the case $\alpha_{1}+\alpha_{2}=0$ of second grade fluids, we obtain regularity results but with severe complications, because of a transport equation which is much more involved than in the simpler case $\alpha_{1}+\alpha_{2}=0$ of second grade fluids.

This paper is organized as follows. The problem and the spaces involved are described in Section 2 . Section 3 is devoted to prove formal a priori estimates satisfied by smooth solutions of the problem and uniqueness of the solution if it exists. Existence is established in Section 4 by applying Galerkin's method with a special basis. The existence and uniqueness results are used in Section 5 to show additional regularity of the solution when the data are also accordingly regular, whence the classical solution. Finally, in Section 6 , we sketch the non-simply-connected case. 


\section{Statement of the PROBlem And NOtATion}

Let $\Omega$ be a bounded domain of $\mathbb{R}^{3}$, simply-connected, with a boundary $\Gamma$ that is at least of class $C^{2,1}$. We denote by $\mathbf{n}$ the unit normal vector to $\Gamma$, directed outside $\Omega$. By setting

$$
p=\tilde{p}-\left(2 \alpha_{1}+\alpha_{2}\right)\left(\mathbf{u} \cdot \Delta \mathbf{u}+\frac{1}{4}\left|A_{1}\right|^{2}\right)+\frac{1}{2}|\mathbf{u}|^{2},
$$

equation (1.5) simplifies and the system of equations we propose to solve is:

Find a vector-valued function $\mathbf{u}=\left(u_{1}, u_{2}, u_{3}\right)$ and a scalar function $p$ defined on $\left.\Omega \times\right] 0, T[$, for some $T>0$, satisfying:

$$
\begin{aligned}
& \frac{\partial}{\partial t}\left(\mathbf{u}-\alpha_{1} \Delta \mathbf{u}\right)-\nu \Delta \mathbf{u}+\operatorname{curl}\left(\mathbf{u}-\left(2 \alpha_{1}+\alpha_{2}\right) \Delta \mathbf{u}\right) \times \mathbf{u}-\left(\alpha_{1}+\alpha_{2}\right) \Delta(\mathbf{u} . \nabla \mathbf{u}) \\
& \left.+2\left(\alpha_{1}+\alpha_{2}\right) \mathbf{u} \cdot \nabla(\Delta \mathbf{u})-\beta \operatorname{div}\left(\left|A_{1}\right|^{2} A_{1}\right)+\nabla p=\mathbf{f} \quad \text { in } \Omega \times\right] 0, T[, \\
& \operatorname{div} \mathbf{u}=0 \quad \text { in } \Omega \times] 0, T[,
\end{aligned}
$$

with homogeneous Dirichlet boundary conditions:

$$
\mathbf{u}=\mathbf{0} \quad \text { on } \Gamma \times] 0, T[,
$$

and initial data:

$$
\mathbf{u}(0)=\mathbf{u}_{0} \quad \text { in } \quad \Omega \text {. }
$$

The parameters $\alpha_{1}, \nu$ and $\beta$ are given positive constants and the initial data $\mathbf{u}_{0}$ satisfies the compatibility condition:

$$
\operatorname{div} \mathbf{u}_{0}=0 \text { in } \Omega \text { and } \mathbf{u}_{0}=\mathbf{0} \text { on } \Gamma .
$$

In order to set this problem into adequate spaces, recall the definition of the following standard Sobolev spaces (cf. [12]). For any multi-index $k=\left(k_{1}, k_{2}, k_{3}\right)$ with $k_{i} \geq 0$, set $|k|=k_{1}+k_{2}+k_{3}$ and denote

$$
\partial^{k} v=\frac{\partial^{|k|} v}{\partial x_{1}^{k_{1}} \partial x_{2}^{k_{2}} \partial x_{3}^{k_{3}}} .
$$

Then for any integer $m \geq 0$ and number $p$ with $1 \leq p \leq \infty$, we define:

$$
W^{m, p}(\Omega)=\left\{v \in L^{p}(\Omega) ; \partial^{k} v \in L^{p}(\Omega) \text { for } 1 \leq|k| \leq m\right\},
$$

which is a Banach space equipped with the norm

$$
\|v\|_{W^{m, p}(\Omega)}=\left(\sum_{|k|=0}^{m} \sum_{k}\left\|\partial^{k} v\right\|_{L^{p}(\Omega)}^{p}\right)^{1 / p},
$$

with the usual modification when $p=\infty$. We denote $H^{m}(\Omega)=W^{m, 2}(\Omega)$.

For vector-valued functions $\mathbf{v}=\left(v_{1}, v_{2}, \ldots, v_{N}\right)$, we use special norms: if $1 \leq p \leq \infty$, we set

$$
\|\mathbf{v}\|_{L^{p}(\Omega)^{N}}=\||\mathbf{v}|\|_{L^{p}(\Omega)},
$$


where $\mid$. | is the Euclidian norm in $\mathbb{R}^{N}$. To simplify, we shall denote $\|\mathbf{v}\|_{L^{p}(\Omega)}$ instead of $\|\mathbf{v}\|_{L^{p}(\Omega)^{N}}$. We consider the matrices $3 \times 3$ as elements of $L^{p}(\Omega)^{9}$ and we define their norms $L^{p}$ by using (2.6) with $N=9$. In the same way, we define the norms of tensors.

We shall frequently use the scalar product of $L^{2}(\Omega)$

$$
(f, g)=\int_{\Omega} f(\mathbf{x}) g(\mathbf{x}) \mathrm{d} \mathbf{x},
$$

the semi-norm of $H^{1}(\Omega)$

$$
|v|_{H^{1}(\Omega)}=\|\nabla v\|_{L^{2}(\Omega)},
$$

and the subspaces of $H^{1}(\Omega), L^{2}(\Omega)^{3}$ and $H^{1}(\Omega)^{3}$ :

$$
\begin{aligned}
H_{0}^{1}(\Omega) & =\left\{v \in H^{1}(\Omega) ; v=0 \text { on } \Gamma\right\}, \\
H(\operatorname{curl} ; \Omega) & =\left\{\mathbf{v} \in L^{2}(\Omega)^{3} ; \operatorname{curl} \mathbf{v} \in L^{2}(\Omega)^{3}\right\}, \\
V & =\left\{\mathbf{v} \in H_{0}^{1}(\Omega)^{3} ; \operatorname{div} \mathbf{v}=0 \text { in } \Omega\right\} .
\end{aligned}
$$

The space $V$ is equipped with the scalar product:

$$
(\mathbf{u}, \mathbf{v})_{V}=(\mathbf{u}, \mathbf{v})+\alpha_{1}(\nabla \mathbf{u}, \nabla \mathbf{v})
$$

and associated norm: $\|\mathbf{v}\|_{V}=(\mathbf{v}, \mathbf{v})_{V}^{1 / 2}$.

Recall also the Poincaré Inequality, valid on any bounded domain: there exists a constant $\mathcal{P}$ such that

$$
\forall v \in H_{0}^{1}(\Omega),\|v\|_{L^{2}(\Omega)} \leq \mathcal{P}|v|_{H^{1}(\Omega)} .
$$

As far as dependence on time is concerned, for any number $T>0$, any Banach space $\mathrm{X}$ and any number $r$ with $1 \leq r \leq \infty$, we define the space

$$
L^{r}(0, T ; X)=\{v:] 0, T\left[\mapsto X ; v \text { is measurable and }\left(\int_{0}^{T}\|v(t)\|_{X}^{r} \mathrm{~d} t\right)^{1 / r}<\infty\right\},
$$

equipped with the norm:

$$
\|v\|_{L^{r}(0, T ; X)}=\left(\int_{0}^{T}\|v(t)\|_{X}^{r} \mathrm{~d} t\right)^{1 / r} .
$$

Following the approach of [5], we introduce the space:

$$
V_{2}=\left\{\mathbf{v} \in V ; \operatorname{curl}\left(\mathbf{v}-\alpha_{1} \Delta \mathbf{v}\right) \in L^{2}(\Omega)^{3}\right\} .
$$

We recall a lemma of [5].

Lemma 2.1. Let $\Omega$ be a bounded, simply-connected open set of $\mathbb{R}^{3}$ with a boundary $\Gamma$ of class $C^{2,1}$. Then any $\mathbf{v}$ in $V_{2}$ belongs to $H^{3}(\Omega)^{3}$ and there exists a constant $C\left(\alpha_{1}\right)$ such that

$$
\forall \mathbf{v} \in V_{2}, \quad\|\mathbf{v}\|_{H^{3}(\Omega)} \leq C\left(\alpha_{1}\right)\left\|\operatorname{curl}\left(\mathbf{v}-\alpha_{1} \Delta \mathbf{v}\right)\right\|_{L^{2}(\Omega)} .
$$


Furthermore, the constant $C\left(\alpha_{1}\right)$ has the bound

$$
C\left(\alpha_{1}\right) \leq \frac{\gamma}{\alpha_{1}} \sqrt{2}, \text { if } \alpha_{1} \geq \frac{1}{2} \text { or } C\left(\alpha_{1}\right) \leq \frac{\gamma}{\alpha_{1}^{3 / 2}}, \text { if } \alpha_{1}<\frac{1}{2},
$$

where $\gamma$ is independent of $\alpha_{1}$.

Remark 2.2. The $C^{2,1}$ regularity of $\Gamma$ is necessary for $\mathbf{v}$ to belong to $H^{3}(\Omega)^{3}$.

Remark 2.3. The argument of Lemma 2.1 can be easily extended to prove that, for any integer $m \geq 0$, if $\Gamma$ is of class $C^{m+2,1}$ and $\mathbf{v}$ belongs to $\mathrm{V}$ with $\operatorname{curl}\left(\mathbf{v}-\alpha_{1} \Delta \mathbf{v}\right)$ in $H^{m}(\Omega)^{3}$, then $\mathbf{v}$ belongs to $H^{m+3}(\Omega)^{3}$ and of course the imbedding is continuous.

According to Lemma 2.1, $V_{2}$ is equipped with the scalar product:

$$
(\mathbf{u}, \mathbf{v})_{V_{2}}=\left(\operatorname{curl}\left(\mathbf{u}-\alpha_{1} \Delta \mathbf{u}\right), \operatorname{curl}\left(\mathbf{v}-\alpha_{1} \Delta \mathbf{v}\right)\right)
$$

and associated norm: $\|\mathbf{v}\|_{V_{2}}=(\mathbf{v}, \mathbf{v})_{V_{2}}^{1 / 2}$. Hence

$$
\forall \mathbf{v} \in V_{2}, \quad\|\mathbf{v}\|_{H^{3}(\Omega)} \leq C\left(\alpha_{1}\right)\|\mathbf{v}\|_{V_{2}} .
$$

We introduce the Sobolev constants $C_{1}$ and $C_{2}$ defined by:

$$
\begin{aligned}
& \forall v \in H^{2}(\Omega), \quad\|v\|_{L^{\infty}(\Omega)} \leq C_{1}\|v\|_{H^{2}(\Omega)} \\
& \forall v \in H^{1}(\Omega), \quad\|v\|_{L^{6}(\Omega)} \leq C_{2}\|v\|_{H^{1}(\Omega)}
\end{aligned}
$$

Then we have with the norms defined by (2.6)

$$
\forall \mathbf{v} \in\left(H^{3}(\Omega)\right)^{N}, \quad\|\nabla \mathbf{v}\|_{L^{\infty}(\Omega)} \leq C_{1}\|\mathbf{v}\|_{H^{3}(\Omega)}
$$

and using Holder's inequality:

$$
\forall \mathbf{v} \in\left(H^{1}(\Omega)\right)^{N}, \quad\|\mathbf{v}\|_{L^{4}(\Omega)} \leq C_{2}^{3 / 4}\|\mathbf{v}\|_{H^{1}(\Omega)}
$$

\section{Variational formulation}

On the one hand, we introduce the trilinear form used in Euler and Navier-Stokes equations:

$$
b(\mathbf{u} ; \mathbf{v}, \mathbf{w})=\sum_{i, j=1}^{3} \int_{\Omega} u_{j} \frac{\partial v_{i}}{\partial x_{j}} w_{i} \mathrm{~d} \mathbf{x} .
$$

On the other hand, we need the following result.

Lemma 2.4. Let $\mathbf{u}$ be given in $H^{3}(\Omega)^{3}$ and $\mathbf{v}$ in $H_{0}^{1}(\Omega)^{3}$. We define the matrix $A(\mathbf{v})$ by:

$$
(A(\mathbf{v}))_{i j}=\frac{\partial v_{i}}{\partial x_{j}}+\frac{\partial v_{j}}{\partial x_{i}}, \quad \text { for } i, j=1,2,3 \text {. }
$$

Then

$$
\left(-\operatorname{div}\left(|A(\mathbf{u})|^{2} A(\mathbf{u})\right), \mathbf{v}\right)=\frac{1}{2}\left(|A(\mathbf{u})|^{2} A(\mathbf{u}), A(\mathbf{v})\right)
$$


Proof. Applying Green's formula, we obtain

$$
\left(-\operatorname{div}\left(|A(\mathbf{u})|^{2} A(\mathbf{u})\right), \mathbf{v}\right)=\int_{\Omega}|A(\mathbf{u})|^{2} \sum_{i, j=1}^{3}\left(\frac{\partial u_{i}}{\partial x_{j}}+\frac{\partial u_{j}}{\partial x_{i}}\right) \frac{\partial v_{i}}{\partial x_{j}} \mathrm{~d} \mathbf{x} .
$$

But

$$
\sum_{i, j=1}^{3}\left(\frac{\partial u_{i}}{\partial x_{j}}+\frac{\partial u_{j}}{\partial x_{i}}\right) \frac{\partial v_{i}}{\partial x_{j}}=\frac{1}{2} \sum_{i, j=1}^{3}\left(\frac{\partial u_{i}}{\partial x_{j}}+\frac{\partial u_{j}}{\partial x_{i}}\right)\left(\frac{\partial v_{i}}{\partial x_{j}}+\frac{\partial v_{j}}{\partial x_{i}}\right) .
$$

Hence, (2.19) follows.

We remark that $A_{1}=A(\mathbf{u})$. Then, owing to the trilinear form $b$ and the previous lemma, we propose the following variational formulation of $(2.1-2.5)$ :

For $\mathbf{f}$ given in $L^{2}(0, T ; H(\mathbf{c u r l} ; \Omega)) \bigcap L^{\infty}\left(0, T ;\left(L^{2}(\Omega)\right)^{3}\right)$ and $\mathbf{u}_{0}$ given in $V_{2}$, find $\mathbf{u}$ in $L^{\infty}\left(0, T ; V_{2}\right)$ with $\mathbf{u}^{\prime}$ in $L^{2}(0, T ; V)$, such that

$$
\begin{aligned}
& \forall \mathbf{v} \in V \quad\left(\mathbf{u}^{\prime}, \mathbf{v}\right)+\alpha_{1}\left(\nabla \mathbf{u}^{\prime}, \nabla \mathbf{v}\right)+\nu(\nabla \mathbf{u}, \nabla \mathbf{v})+\left(\operatorname{curl}\left(\mathbf{u}-\left(2 \alpha_{1}+\alpha_{2}\right) \Delta \mathbf{u}\right) \times \mathbf{u}, \mathbf{v}\right) \\
& \quad+\left(\alpha_{1}+\alpha_{2}\right)[(\nabla(\mathbf{u} . \nabla \mathbf{u}), \nabla \mathbf{v})+2 b(\mathbf{u} ; \Delta \mathbf{u}, \mathbf{v})]+\frac{\beta}{2}\left(|A(\mathbf{u})|^{2} A(\mathbf{u}), A(\mathbf{v})\right)=(\mathbf{f}, \mathbf{v})
\end{aligned}
$$

with the initial condition (2.4).

Clearly, by restricting the set of solutions of $(2.1-2.5)$ to $L^{\infty}\left(0, T ; V_{2}\right)$ with the first derivative in $L^{2}(0, T ; V)$, this formulation is equivalent to $(2.1-2.5)$.

As mentioned in the introduction, we shall obtain a transport equation by taking the curl of equation (2.1). Before, we require the following technical results.

Lemma 2.5. Formally, we have

$$
\operatorname{curl}(\mathbf{u} . \nabla \mathbf{v})=\mathbf{u} . \nabla \operatorname{curl} \mathbf{v}-\operatorname{curl} \mathbf{v} . \nabla \mathbf{u}+\operatorname{div} \mathbf{u} \operatorname{curl} \mathbf{v}+\sum_{k=1}^{3} \nabla u_{k} \times \nabla v_{k} .
$$

Proof. We can verify this identity for each component.

Lemma 2.6. We denote $A_{., j}$ the $j$ th column vector of the matrix $A$. Suppose that $\operatorname{div} \mathbf{v}=0$. Formally, we have

$$
\operatorname{curl}\left(\operatorname{div}\left(|A(\mathbf{v})|^{2} A(\mathbf{v})\right)\right)=|A(\mathbf{v})|^{2} \Delta(\operatorname{curl} \mathbf{v})+2 \nabla\left(|A(\mathbf{v})|^{2}\right) \cdot \nabla \operatorname{curl} \mathbf{v}+\mathbf{B}(\mathbf{v})
$$

where

$$
\mathbf{B}(\mathbf{v})=\sum_{k=1}^{3}\left[\nabla\left(\frac{\partial}{\partial x_{k}}\left(|A(\mathbf{v})|^{2}\right)\right) \times A_{., k}(\mathbf{v})-\frac{\partial}{\partial x_{k}}\left(|A(\mathbf{v})|^{2}\right) \nabla\left((\mathbf{c u r l} \mathbf{v})_{k}\right)\right]
$$

Proof. We can verify, using the identity: $\operatorname{div}(\theta \mathbf{v})=\theta \operatorname{div} \mathbf{v}+\nabla \theta \cdot \mathbf{v}$,

$$
\operatorname{div}\left(|A(\mathbf{v})|^{2} A(\mathbf{v})\right)=|A(\mathbf{v})|^{2} \Delta \mathbf{v}+\sum_{k=1}^{3} \frac{\partial}{\partial x_{k}}\left(|A(\mathbf{v})|^{2}\right) A_{., k}(\mathbf{v}) .
$$


Next, we have, owing mainly to the identity: $\operatorname{curl}(\theta \mathbf{v})=\theta \operatorname{curl} \mathbf{v}+\nabla \theta \times \mathbf{v}$,

$$
\operatorname{curl}\left(|A(\mathbf{v})|^{2} \Delta \mathbf{v}\right)=|A(\mathbf{v})|^{2} \Delta(\operatorname{curl} \mathbf{v})+\nabla\left(|A(\mathbf{v})|^{2}\right) . \nabla \operatorname{curl} \mathbf{v}-\sum_{k=1}^{3} \frac{\partial}{\partial x_{k}}\left(|A(\mathbf{v})|^{2}\right) \nabla\left((\operatorname{curl} \mathbf{v})_{k}\right)
$$

and

$$
\operatorname{curl}\left(\sum_{k=1}^{3} \frac{\partial}{\partial x_{k}}\left(|A(\mathbf{v})|^{2}\right) A_{., k}(\mathbf{v})\right)=\nabla\left(|A(\mathbf{v})|^{2}\right) . \nabla \operatorname{curl} \mathbf{v}+\sum_{k=1}^{3} \nabla\left(\frac{\partial}{\partial x_{k}}\left(|A(\mathbf{v})|^{2}\right)\right) \times A_{., k}(\mathbf{v}) .
$$

From $(2.24,2.25,2.26)$, we derive $(2.22)$.

Set $\boldsymbol{\omega}=\mathbf{c u r l} \mathbf{u}$. From Lemma 2.5 and $\operatorname{div} \mathbf{u}=0$, it follows that:

$$
\operatorname{curl}(\mathbf{u} . \nabla(\Delta \mathbf{u}))=\mathbf{u} \cdot \nabla(\Delta \boldsymbol{\omega})-\Delta \boldsymbol{\omega} \cdot \nabla \mathbf{u}+\sum_{k=1}^{3} \nabla u_{k} \times \nabla\left(\Delta u_{k}\right)
$$

and

$$
\operatorname{curl}(\Delta(\mathbf{u} \cdot \nabla \mathbf{u}))=\Delta \mathbf{u} \cdot \nabla \boldsymbol{\omega}+\mathbf{u} \cdot \nabla(\Delta \boldsymbol{\omega})-\Delta \boldsymbol{\omega} \cdot \nabla \mathbf{u}-\boldsymbol{\omega} \cdot \nabla(\Delta \mathbf{u})+2 \sum_{k=1}^{3}\left(\frac{\partial \mathbf{u}}{\partial x_{k}} \cdot \nabla \frac{\partial \boldsymbol{\omega}}{\partial x_{k}}-\frac{\partial \boldsymbol{\omega}}{\partial x_{k}} \cdot \nabla \frac{\partial \mathbf{u}}{\partial x_{k}}\right) .
$$

Finally,

$$
\operatorname{curl}\left(\operatorname{curl}\left(\mathbf{u}-\left(2 \alpha_{1}+\alpha_{2}\right) \Delta \mathbf{u}\right) \times \mathbf{u}\right)=\mathbf{u} \cdot \nabla\left(\boldsymbol{\omega}-\left(2 \alpha_{1}+\alpha_{2}\right) \Delta \boldsymbol{\omega}\right)-\left(\boldsymbol{\omega}-\left(2 \alpha_{1}+\alpha_{2}\right) \Delta \boldsymbol{\omega}\right) . \nabla \mathbf{u} .
$$

Thus, we derive formally from $(2.27-2.29,2.22)$ the following transport equation:

$$
\begin{aligned}
\frac{\partial}{\partial t}\left(\boldsymbol{\omega}-\alpha_{1} \Delta \boldsymbol{\omega}\right) & \frac{\nu}{\alpha_{1}}\left(\boldsymbol{\omega}-\alpha_{1} \Delta \boldsymbol{\omega}\right)+\mathbf{u} \cdot \nabla\left(\boldsymbol{\omega}-\alpha_{1} \Delta \boldsymbol{\omega}\right)-\left(\boldsymbol{\omega}-\alpha_{1} \Delta \boldsymbol{\omega}\right) . \nabla \mathbf{u}-\left(\alpha_{1}+\alpha_{2}\right) \Delta \mathbf{u} . \nabla \boldsymbol{\omega} \\
& +\left(\alpha_{1}+\alpha_{2}\right)\left[\boldsymbol{\omega} \cdot \nabla(\Delta \mathbf{u})+2 \sum_{k=1}^{3}\left(\frac{\partial \boldsymbol{\omega}}{\partial x_{k}} \cdot \nabla \frac{\partial \mathbf{u}}{\partial x_{k}}-\frac{\partial \mathbf{u}}{\partial x_{k}} \cdot \nabla \frac{\partial \boldsymbol{\omega}}{\partial x_{k}}+\nabla u_{k} \times \nabla \Delta u_{k}\right)\right]+\beta[-\mathbf{B}(\mathbf{u}) \\
+ & \left.\frac{1}{\alpha_{1}}|A(\mathbf{u})|^{2}\left(\boldsymbol{\omega}-\alpha_{1} \Delta \boldsymbol{\omega}\right)-2 \nabla\left(|A(\mathbf{u})|^{2}\right) . \nabla \boldsymbol{\omega}\right]=\mathbf{c u r l} \mathbf{f}+\frac{\nu}{\alpha_{1}} \operatorname{curl} \mathbf{u}+\frac{\beta}{\alpha_{1}}|A(\mathbf{u})|^{2} \mathbf{c u r l} \mathbf{u} .
\end{aligned}
$$

This equation has to be interpreted in the sense of distributions, unless $\mathbf{u}(t)$ belongs to $H^{4}(\Omega)^{3}$.

\section{A PRIORI ESTIMATES AND UNIQUENESS}

The a priori estimates of this section are formal because they are derived for the exact solution of problem $(2.20,2.4)$, whose regularity is not known. However, in the next section, we shall apply these estimates rigorously to the solution of the Galerkin approximation of problem (2.20), and we know from the onset that this solution is sufficiently smooth.

Lemma 3.1. Suppose problem (2.20, 2.4) has a solution $\mathbf{u}$ in $C^{0}\left(0, T ; V_{2}\right)$ with $\mathbf{u}^{\prime}$ in $L^{\infty}(0, T ; V)$. Set

$$
K_{1}=\frac{\nu}{2\left(\mathcal{P}^{2}+\alpha_{1}\right)}, K_{2}=\frac{6}{\alpha_{1}}\left|\alpha_{1}+\alpha_{2}\right| C_{1} C\left(\alpha_{1}\right) .
$$


Then this solution satisfies the following inequalities for all t in [0,T]:

$$
\begin{aligned}
\|\mathbf{u}(t)\|_{V}^{2} \leq & \mathrm{e}^{-K_{1} t}\left\|\mathbf{u}_{0}\right\|_{V}^{2}+\frac{\mathcal{P}^{2}}{\nu} \int_{0}^{t} \mathrm{e}^{-K_{1}(t-s)}\|\mathbf{f}(s)\|_{L^{2}(\Omega)}^{2} \mathrm{~d} s \\
& -K_{2} \int_{0}^{t} \mathrm{e}^{-K_{1}(t-s)}\left(\frac{K_{1}}{K_{2}}-\|\mathbf{u}(s)\|_{V_{2}}\right)\|\mathbf{u}(s)\|_{V}^{2} \mathrm{~d} s \\
\int_{0}^{t}\||A(\mathbf{u}(s))|\|_{L^{4}(\Omega)}^{4} \mathrm{~d} s \leq & \left.\frac{1}{\beta}\left(\left\|\mathbf{u}_{0}\right\|_{V}^{2}+\frac{\mathcal{P}^{2}}{\nu} \int_{0}^{t}\|\mathbf{f}(s)\|_{L^{2}(\Omega)}^{2} \mathrm{~d} s\right)-\frac{K_{2}}{\beta} \int_{0}^{t}\left(\frac{K_{1}}{K_{2}}-\|\mathbf{u}(s)\|_{V_{2}}\right)\|\mathbf{u}(s)\|_{V}^{2} \mathrm{~d} s\right) .
\end{aligned}
$$

Proof. The choice $\mathbf{v}=\mathbf{u}$ in $(2.20)$, the anti-symmetry of $b$, the relation $(\mathbf{w} \times \mathbf{v}, \mathbf{v})=0$ and the definition $(2.7)$ imply

$$
\frac{1}{2} \frac{\mathrm{d}}{\mathrm{d} t}\|\mathbf{u}(t)\|_{V}^{2}+\nu|\mathbf{u}(t)|_{H^{1}(\Omega)}^{2}+3\left(\alpha_{1}+\alpha_{2}\right) b(\mathbf{u}(t) ; \Delta \mathbf{u}(t), \mathbf{u}(t))+\frac{\beta}{2}\||A(\mathbf{u}(t))|\|_{L^{4}(\Omega)}^{4}=(\mathbf{f}(t), \mathbf{u}(t)) .
$$

Observing that

$$
\forall \mathbf{v} \in H_{0}^{1}(\Omega)^{3}, \quad \frac{1}{\mathcal{P}^{2}+\alpha_{1}}\|\mathbf{v}\|_{V}^{2} \leq|\mathbf{v}|_{H^{1}(\Omega)}^{2} \leq \frac{1}{\alpha_{1}}\|\mathbf{v}\|_{V}^{2},
$$

we obtain

$$
\frac{1}{2} \frac{\mathrm{d}}{\mathrm{d} t}\|\mathbf{u}(t)\|_{V}^{2}+\frac{\nu}{\mathcal{P}^{2}+\alpha_{1}}\|\mathbf{u}(t)\|_{V}^{2}+\frac{\beta}{2}\||A(\mathbf{u}(t))|\|_{L^{4}(\Omega)}^{4} \leq|(\mathbf{f}(t), \mathbf{u}(t))|+3\left|\alpha_{1}+\alpha_{2} \| b(\mathbf{u}(t) ; \Delta \mathbf{u}(t), \mathbf{u}(t))\right| .
$$

Since $\mathbf{v}$ belongs to $V_{2}$, we derive by applications of Green's formula that

$$
b(\mathbf{v} ; \Delta \mathbf{v}, \mathbf{v})=\sum_{i, j, k=1}^{3} \int_{\Omega} v_{k} \frac{\partial^{3} v_{i}}{\partial x_{k} \partial x_{j}^{2}} v i \mathrm{~d} \mathbf{x}=\sum_{i, j, k=1}^{3} \int_{\Omega} \frac{\partial v_{k}}{\partial x_{j}} \frac{\partial v_{i}}{\partial x_{j}} \frac{\partial v_{i}}{\partial x_{k}} \mathrm{~d} \mathbf{x}
$$

hence

$$
|b(\mathbf{u}(t) ; \Delta \mathbf{u}(t), \mathbf{u}(t))| \leq\left\|\left(\sum_{j, k=1}^{3} \frac{\partial u_{k}(t)^{2}}{\partial x_{j}}\right)^{1 / 2}\right\|_{L^{\infty}(\Omega)} \int_{\Omega} \sum_{i=1}^{3}\left(\sum_{j, k=1}^{3} \frac{\partial u_{i}(t)^{2}}{\partial x_{j}} \frac{\partial u_{i}(t)^{2}}{\partial x_{k}}\right)^{1 / 2} \mathrm{~d} \mathbf{x} .
$$

Then $(2.16,2.13,3.4)$ imply

$$
|b(\mathbf{u}(t) ; \Delta \mathbf{u}(t), \mathbf{u}(t))| \leq\|\nabla \mathbf{u}(t)\|_{L^{\infty}(\Omega)}|\mathbf{u}(t)|_{H^{1}(\Omega)}^{2} \leq \frac{C_{1} C\left(\alpha_{1}\right)}{\alpha_{1}}\|\mathbf{u}(t)\|_{V_{2}}\|\mathbf{u}(t)\|_{V}^{2}
$$

Moreover, we have

$$
|(\mathbf{f}(t), \mathbf{u}(t))| \leq \frac{\mathcal{P}^{2}}{2 \nu}\|\mathbf{f}(t)\|_{L^{2}(\Omega)}^{2}+\frac{\nu}{2}|\mathbf{u}(t)|_{H^{1}(\Omega)}^{2} .
$$

By substituting into (3.5), simplifying by $\|\mathbf{u}(t)\|_{V}$ and using the constants $K_{1}$ and $K_{2}$, we obtain for all $t$ in $[0, T]$ :

$$
\frac{\mathrm{d}}{\mathrm{d} t}\|\mathbf{u}(t)\|_{V}^{2}+K_{1}\|\mathbf{u}(t)\|_{V}^{2}+\beta\||A(\mathbf{u}(t))|\|_{L^{4}(\Omega)}^{4} \leq \frac{\mathcal{P}^{2}}{\nu}\|\mathbf{f}(t)\|_{L^{2}(\Omega)}^{2}-K_{2}\left(\frac{K_{1}}{K_{2}}-\|\mathbf{u}(t)\|_{V_{2}}\right)\|\mathbf{u}(t)\|_{V}^{2} .
$$


Then (3.1) is derived by multiplying both sides of (3.8) by $e^{K_{1} t}$ and integrating over $[0, t]$. At last, we obtain (3.2) by integrating $(3.8)$ over $[0, t]$.

Remark 3.2. The term $b(\mathbf{u}(t) ; \Delta \mathbf{u}(t), \mathbf{u}(t))$ gives rise to the factor $\|\mathbf{u}(t)\|_{V_{2}}$ in the right-hand side of $(3.1)$. Therefore (3.1) alone does not give an estimate for $\|\mathbf{u}(t)\|_{V}$. We shall complete (3.1) with an estimate derived from the transport equation that will upbound simultaneously $\|\mathbf{u}(t)\|_{V}$ and $\|\mathbf{u}(t)\|_{V_{2}}$ for small data.

Theorem 3.3. In addition to the assumptions of Lemma 3.1, suppose that $\operatorname{curl}(\Delta \mathbf{u})$ belongs to $L^{2}\left(0, T ; H^{1}(\Omega)^{3}\right)$. Then $y(t)=\|\mathbf{u}(t)\|_{V_{2}}^{2}$ satisfies the differential inequality in [0,T]:

$$
\begin{aligned}
y^{\prime}(t) \leq & \frac{4 \nu}{\alpha_{1}^{2}}\left(\mathrm{e}^{-K_{1} t}\left\|\mathbf{u}_{0}\right\|_{V}^{2}+\frac{\mathcal{P}^{2}}{\nu} \int_{0}^{t} \mathrm{e}^{-K_{1}(t-s)}\|\mathbf{f}(s)\|_{L^{2}(\Omega)}^{2} \mathrm{~d} s\right) \\
& +\frac{2 \alpha_{1}}{\nu}\|\mathbf{c u r l} \mathbf{f}(t)\|_{L^{2}(\Omega)}^{2}-C\left(\alpha_{1}, \alpha_{2}, \beta\right)\left(\frac{\nu}{2 \alpha_{1} C\left(\alpha_{1}, \alpha_{2}, \beta\right)}-y(t)\right) y(t) \\
& +\frac{\beta C\left(\alpha_{1}\right)}{2 \alpha_{1}^{2}}\||A(\mathbf{u}(t))|\|_{L^{4}(\Omega)}^{4}-\frac{4 \nu}{\alpha_{1}^{2}} K_{2} \int_{0}^{t} \mathrm{e}^{-K_{1}(t-s)}\left(\frac{K_{1}}{K_{2}}-\sqrt{y(s)}\right)\|\mathbf{u}(s)\|_{V}^{2} \mathrm{~d} s,
\end{aligned}
$$

where

$$
C\left(\alpha_{1}, \alpha_{2}, \beta\right)=\frac{2 \alpha_{1}}{\nu}\left(C\left(\alpha_{1}, \alpha_{2}\right)\right)^{2}+\beta\left(C\left(\alpha_{1}\right)\right)^{3}\left(16 C_{1}\left((3 \sqrt{2}+2) C_{2}^{3 / 2}+2 C_{1}\right)+\frac{C_{2}^{3}}{2}\right)
$$

with $C\left(\alpha_{1}, \alpha_{2}\right)=C_{1} C\left(\alpha_{1}\right)+\left|\alpha_{1}+\alpha_{2}\right|\left(C\left(\alpha_{1}\right)\right)^{2}\left[(\sqrt{6}+2(\sqrt{3}+\sqrt{2})) C_{1}+(\sqrt{6}+2 \sqrt{2}) C_{2}^{3 / 2}\right]$

and $K_{1}$ and $K_{2}$ are defined in Lemma 3.1 .

Proof. To simplify, set $\mathbf{z}=\boldsymbol{\omega}-\alpha_{1} \Delta \boldsymbol{\omega}$. Then taking the scalar product of (2.30) with $\mathbf{z}$ gives:

$$
\begin{aligned}
\frac{1}{2} \frac{\mathrm{d}}{\mathrm{d} t}\|\mathbf{u}(t)\|_{V_{2}}^{2}+ & \frac{\nu}{\alpha_{1}}\|\mathbf{u}(t)\|_{V_{2}}^{2}-b(\mathbf{z}(t) ; \mathbf{u}(t), \mathbf{z}(t))+\left(\alpha_{1}+\alpha_{2}\right)\{-b(\Delta \mathbf{u}(t) ; \boldsymbol{\omega}(t), \mathbf{z}(t)) \\
& +b(\boldsymbol{\omega}(t) ; \Delta \mathbf{u}(t), \mathbf{z}(t))+2 \sum_{k=1}^{3}\left[b\left(\frac{\partial \boldsymbol{\omega}(t)}{\partial x_{k}} ; \frac{\partial \mathbf{u}(t)}{\partial x_{k}}, \mathbf{z}(t)\right)-b\left(\frac{\partial \mathbf{u}(t)}{\partial x_{k}} ; \frac{\partial \boldsymbol{\omega}(t)}{\partial x_{k}}, \mathbf{z}(t)\right)\right. \\
+ & \left.\left.\left(\nabla u_{k}(t) \times \nabla \Delta u_{k}(t), \mathbf{z}(t)\right)\right]\right\}-\beta\left\{2\left(\nabla\left(|A(\mathbf{u}(t))|^{2}\right) . \nabla \boldsymbol{\omega}(t), \mathbf{z}(t)\right)+(\mathbf{B}(\mathbf{u}(t)), \mathbf{z}(t))\right\} \\
& +\frac{\beta}{\alpha_{1}}\||A(\mathbf{u}(t))| \mathbf{z}(t)\|_{L^{2}(\Omega)}^{2}=\left(\operatorname{curl}\left(\mathbf{f}(t)+\frac{\nu}{\alpha_{1}} \mathbf{u}(t)\right), \mathbf{z}(t)\right)+\frac{\beta}{\alpha_{1}}\left(|A(\mathbf{u}(t))|^{2} \mathbf{c u r l} \mathbf{u}(t), \mathbf{z}(t)\right) .
\end{aligned}
$$

Considering that, for all $\mathbf{u}$ and $\mathbf{w}$ in $L^{2}(\Omega)^{3}$ and all $\mathbf{v}$ in $W^{1, \infty}(\Omega)^{3}$,

$$
|b(\mathbf{u} ; \mathbf{v}, \mathbf{w})| \leq\|\mathbf{u}\|_{L^{2}(\Omega)}\|\nabla \mathbf{v}\|_{L^{\infty}(\Omega)}\|\mathbf{w}\|_{L^{2}(\Omega)},
$$

$(2.16,2.13)$ yield

$$
|b(\mathbf{z}(t) ; \mathbf{u}(t), \mathbf{z}(t))| \leq\|\nabla \mathbf{u}(t)\|_{L^{\infty}(\Omega)}\|\mathbf{z}(t)\|_{L^{2}(\Omega)}^{2} \leq C_{1} C\left(\alpha_{1}\right)\|\mathbf{u}(t)\|_{V_{2}}^{3} .
$$

Next, considering that for all $\mathbf{u}$ in $L^{4}(\Omega)^{3}, \mathbf{v}$ in $W^{1,4}(\Omega)^{3}$ and $\mathbf{w}$ in $L^{2}(\Omega)^{3}$,

$$
|b(\mathbf{u} ; \mathbf{v}, \mathbf{w})| \leq\|\mathbf{u}\|_{L^{4}(\Omega)}\|\nabla \mathbf{v}\|_{L^{4}(\Omega)}\|\mathbf{w}\|_{L^{2}(\Omega)},
$$

$(2.17,2.13)$ gives

$$
\begin{aligned}
|b(\Delta \mathbf{u}(t) ; \boldsymbol{\omega}(t), \mathbf{z}(t))| & \leq\|\Delta \mathbf{u}(t)\|_{L^{4}(\Omega)}\|\nabla \boldsymbol{\omega}(t)\|_{L^{4}(\Omega)}\|\mathbf{z}(t)\|_{L^{2}(\Omega)} \\
& \leq \sqrt{6} C_{2}^{3 / 2}\left(C\left(\alpha_{1}\right)\right)^{2}\|\mathbf{u}(t)\|_{V_{2}}^{3} .
\end{aligned}
$$


By similar argument, we obtain

$$
\begin{aligned}
|b(\boldsymbol{\omega}(t) ; \Delta \mathbf{u}(t), \mathbf{z}(t))| & \leq\|\boldsymbol{\omega}(t)\|_{L^{\infty}(\Omega)}|\Delta \mathbf{u}(t)|_{H^{1}(\Omega)}\|\mathbf{z}(t)\|_{L^{2}(\Omega)} \leq \sqrt{6} C_{1}\left(C\left(\alpha_{1}\right)\right)^{2}\|\mathbf{u}(t)\|_{V_{2}}^{3} \\
\left|\sum_{k=1}^{3} b\left(\frac{\partial \mathbf{u}(t)}{\partial x_{k}} ; \frac{\partial \boldsymbol{\omega}(t)}{\partial x_{k}}, \mathbf{z}(t)\right)\right| & \leq \sqrt{2} C_{1}\left(C\left(\alpha_{1}\right)\right)^{2}\|\mathbf{u}(t)\|_{V_{2}}^{3}, \\
\left|\sum_{k=1}^{3} b\left(\frac{\partial \boldsymbol{\omega}(t)}{\partial x_{k}} ; \frac{\partial \mathbf{u}(t)}{\partial x_{k}}, \mathbf{z}(t)\right)\right| & \leq \sqrt{2} C_{2}^{3 / 2}\left(C\left(\alpha_{1}\right)\right)^{2}\|\mathbf{u}(t)\|_{V_{2}}^{3}
\end{aligned}
$$

and

$$
\left|\sum_{k=1}^{3}\left(\nabla u_{k}(t) \times \nabla \Delta u_{k}(t), \mathbf{z}(t)\right)\right| \leq \sqrt{3} C_{1}\left(C\left(\alpha_{1}\right)\right)^{2}\|\mathbf{u}(t)\|_{V_{2}}^{3} .
$$

Considering that

$$
|A(\mathbf{u})|^{2} \leq 4 \sum_{i, j=1}^{3}\left(\frac{\partial u_{i}}{\partial x_{j}}\right)^{2} \text { and } \sum_{i, j, k=1}^{3}\left(\frac{\partial A_{i j}(\mathbf{u})}{\partial x_{k}}\right)^{2} \leq 4 \sum_{i, j, k=1}^{3}\left(\frac{\partial^{2} u_{i}}{\partial x_{j} \partial x_{k}}\right)^{2},
$$

by using Hölder, Cauchy-Schwarz, $(2.17,2.16,2.13)$, we obtain

$$
\begin{aligned}
\left|\left(\nabla\left(|A(\mathbf{u})|^{2}\right) . \nabla \boldsymbol{\omega}, \mathbf{z}\right)\right| & \leq 2\|\mathbf{z}\|_{L^{2}(\Omega)}\||A(\mathbf{u})|\|_{L^{\infty}(\Omega)}\left[\int_{\Omega}\left(\sum_{k, l=1}^{3}\left(\frac{\partial \omega_{l}}{\partial x_{k}}\right)^{2}\right)\left(\sum_{i, j, k=1}^{3}\left(\frac{\partial A_{i j}(\mathbf{u})}{\partial x_{k}}\right)^{2}\right) \mathrm{d} \mathbf{x}\right]^{1 / 2} \\
& \leq 8 \sqrt{2} C_{1} C_{2}^{3 / 2}\left(C\left(\alpha_{1}\right)\right)^{3}\|\mathbf{u}(t)\|_{V_{2}}^{4} .
\end{aligned}
$$

By expanding the two terms of $\mathbf{B}(\mathbf{u})$ and by using the same techniques, we have

$$
\left|\left(\sum_{k=1}^{3} \nabla\left(\frac{\partial}{\partial x_{k}}|A(\mathbf{u}(t))|^{2}\right) \times A_{., k}(\mathbf{u}(t)), \mathbf{z}(t)\right)\right| \leq 16 C_{1}\left(C_{1}+C_{2}^{3 / 2}\right)\left(C\left(\alpha_{1}\right)\right)^{3}\|\mathbf{u}(t)\|_{V_{2}}^{4}
$$

and

$$
\left|\left(\sum_{k=1}^{3} \frac{\partial}{\partial x_{k}}\left(|A(\mathbf{u}(t))|^{2}\right) \nabla \omega_{k}(t), \mathbf{z}(t)\right)\right| \leq 8 \sqrt{2} C_{1} C_{2}^{3 / 2}\left(C\left(\alpha_{1}\right)\right)^{3}\|\mathbf{u}(t)\|_{V_{2}}^{4} .
$$

Bounding the right side of (3.11) in the same way as (3.7) and collecting the inequalities (3.12-3.20), we derive

$$
\begin{aligned}
\frac{\mathrm{d}}{\mathrm{d} t}\|\mathbf{u}(t)\|_{V_{2}}^{2}+\frac{\nu}{\alpha_{1}}\|\mathbf{u}(t)\|_{V_{2}}^{2} \leq & \frac{2 \alpha_{1}}{\nu}\|\mathbf{c u r l} \mathbf{f}(t)\|_{L^{2}(\Omega)}^{2}+\frac{2 \nu}{\alpha_{1}}\|\mathbf{c u r l} \mathbf{u}(t)\|_{L^{2}(\Omega)}^{2}+2 C\left(\alpha_{1}, \alpha_{2}\right)\|\mathbf{u}(t)\|_{V_{2}}^{3} \\
& +16 \beta C_{1}\left((3 \sqrt{2}+2) C_{2}^{3 / 2}+2 C_{1}\right)\left(C\left(\alpha_{1}\right)\right)^{3}\|\mathbf{u}(t)\|_{V_{2}}^{4} \\
& +\frac{\beta}{2 \alpha_{1}}\||A(\mathbf{u}(t))| \operatorname{curl} \mathbf{u}(t)\|_{L^{2}(\Omega)}^{2} .
\end{aligned}
$$

Now, using the bound

$$
\||A(\mathbf{u}(t))| \operatorname{curl} \mathbf{u}(t)\|_{L^{2}(\Omega)}^{2} \leq \frac{C\left(\alpha_{1}\right)}{\alpha_{1}}\||A(\mathbf{u}(t))|\|_{L^{4}(\Omega)}^{4}+\alpha_{1}\left(C\left(\alpha_{1}\right)\right)^{3} C_{2}^{3}\|\mathbf{u}(t)\|_{V_{2}}^{4}
$$


and setting $y(t)=\|\mathbf{u}(t)\|_{V_{2}}^{2},(3.21)$ implies

$$
\begin{aligned}
y^{\prime}(t)+\frac{\nu}{\alpha_{1}} y(t) \leq & \frac{2 \alpha_{1}}{\nu}\|\mathbf{c u r l} \mathbf{f}(t)\|_{L^{2}(\Omega)}^{2}+\frac{4 \nu}{\alpha_{1}^{2}}\|\mathbf{u}(t)\|_{V}^{2}+2 C\left(\alpha_{1}, \alpha_{2}\right) y(t) \sqrt{y(t)} \\
& +\beta\left(C\left(\alpha_{1}\right)\right)^{3}\left(16 C_{1}\left((3 \sqrt{2}+2) C_{2}^{3 / 2}+2 C_{1}\right)+\frac{C_{2}^{3}}{2}\right)(y(t))^{2}+\frac{\beta C\left(\alpha_{1}\right)}{2 \alpha_{1}^{2}} \| \mid A\left(\mathbf{u}(t) \mid \|_{L^{4}(\Omega)}^{4} .\right.
\end{aligned}
$$

Finally, considering that $\sqrt{y} \leq 1 / 4 \theta+\theta$, we obtain

$$
\begin{aligned}
y^{\prime}(t) \leq & \frac{2 \alpha_{1}}{\nu}\|\mathbf{c u r l} \mathbf{f}(t)\|_{L^{2}(\Omega)}^{2}+\frac{4 \nu}{\alpha_{1}^{2}}\|\mathbf{u}(t)\|_{V}^{2} \\
& +\frac{\beta C\left(\alpha_{1}\right)}{2 \alpha_{1}^{2}} \| \mid A\left(\mathbf{u}(t) \mid \|_{L^{4}(\Omega)}^{4}-C\left(\alpha_{1}, \alpha_{2}, \beta\right)\left(\frac{\nu}{2 \alpha_{1} C\left(\alpha_{1}, \alpha_{2}, \beta\right)}-y(t)\right) y(t),\right.
\end{aligned}
$$

and we derive (3.9) by substituting (3.1) in this inequality.

In the absence of additional information on the solution $\mathbf{u}$, it is clear from the differential inequality (3.9) that we cannot prove global existence in time of $\mathbf{u}$ unless we show the uniform bound:

$$
\forall t>0,0 \leq y(t) \leq \min \left(\frac{\nu}{2 \alpha_{1} C\left(\alpha_{1}, \alpha_{2}, \beta\right)}, \frac{K_{1}^{2}}{K_{2}^{2}}\right)
$$

Owing to the exponential decay of terms of (3.9), we shall prove that every continuous solution of (3.9) satisfies (3.22) for small enough data.

Lemma 3.4. Let $\mathbf{f}$ belong to $L^{2}\left(\mathbb{R}^{+} ; H(\mathbf{c u r l} ; \Omega)\right)$. If the data satisfy:

$$
\begin{aligned}
\left\|\mathbf{u}_{0}\right\|_{V_{2}}^{2}+\frac{8 \nu+C\left(\alpha_{1}\right) K_{1}}{2 \alpha_{1}^{2} K_{1}}\left(\left\|\mathbf{u}_{0}\right\|_{V}^{2}+\frac{\mathcal{P}^{2}}{\nu} \int_{0}^{\infty}\|\mathbf{f}(t)\|_{L^{2}(\Omega)}^{2} \mathrm{~d} t\right)+\frac{2 \alpha_{1}}{\nu} \int_{0}^{\infty} & \|\operatorname{curl} \mathbf{f}(t)\|_{L^{2}(\Omega)}^{2} \mathrm{~d} t \\
& <\min \left(\frac{\nu}{2 \alpha_{1} C\left(\alpha_{1}, \alpha_{2}, \beta\right)}, \frac{K_{1}^{2}}{K_{2}^{2}}\right)
\end{aligned}
$$

where $C\left(\alpha_{1}, \alpha_{2}, \beta\right)$ is defined by (3.10) and $K_{1}$ and $K_{2}$ are defined as in Lemma 3.1, then any continuous solution of (3.9) with starting value $y(0)=\left\|\mathbf{u}_{0}\right\|_{V_{2}}^{2}$ satisfies:

$$
\forall t \geq 0,0 \leq y(t) \leq \min \left(\frac{\nu}{2 \alpha_{1} C\left(\alpha_{1}, \alpha_{2}, \beta\right)}, \frac{K_{1}^{2}}{K_{2}^{2}}\right)
$$

Proof. Let us integrate (3.9) from 0 to $t$. Applying the following result due to Fubini:

$$
\forall t \geq 0, \forall h \in L^{1}\left(\mathbb{R}^{+}\right), \forall A>0, \int_{0}^{t}\left(\int_{0}^{s} \mathrm{e}^{-A(s-\tau)} h(\tau) \mathrm{d} \tau\right) \mathrm{d} s=\frac{1}{A} \int_{0}^{t} h(s)\left(1-\mathrm{e}^{-A(t-s)}\right) \mathrm{d} s
$$


and substituting the inequality (3.2), we derive

$$
\begin{aligned}
y(t) \leq & y(0)+\frac{8 \nu+C\left(\alpha_{1}\right) K_{1}}{2 K_{1} \alpha_{1}^{2}}\left(\left\|\mathbf{u}_{0}\right\|_{V}^{2}+\frac{\mathcal{P}^{2}}{\nu} \int_{0}^{t}\|\mathbf{f}(s)\|_{L^{2}(\Omega)}^{2} \mathrm{~d} s\right) \\
& +\frac{2 \alpha_{1}}{\nu} \int_{0}^{t}\|\mathbf{c u r l} \mathbf{f}(s)\|_{L^{2}(\Omega)}^{2} \mathrm{~d} s-C\left(\alpha_{1}, \alpha_{2}, \beta\right) \int_{0}^{t}\left(\frac{\nu}{2 \alpha_{1} C\left(\alpha_{1}, \alpha_{2}, \beta\right)}-y(s)\right) y(s) \mathrm{d} s \\
& -\frac{K_{2}}{2 \alpha_{1}^{2}} \int_{o}^{t}\left(\frac{K_{1}}{K_{2}}-\sqrt{y(s)}\right)\|\mathbf{u}(s)\|_{V}^{2}\left[C\left(\alpha_{1}\right)+16\left(\mathcal{P}^{2}+\alpha_{1}\right)\left(1-\mathrm{e}^{-K_{1}(t-s)}\right)\right] \mathrm{d} s .
\end{aligned}
$$

We set $M=\min \left(\frac{\nu}{2 \alpha_{1} C\left(\alpha_{1}, \alpha_{2}, \beta\right)}, \frac{K_{1}^{2}}{K_{2}^{2}}\right)$ and

$$
a(s, t)=C\left(\alpha_{1}, \alpha_{2}, \beta\right) y(s)+\frac{K_{2}^{2}}{2 \alpha_{1}^{2}\left(K_{1}+K_{2} \sqrt{y(s)}\right)}\|\mathbf{u}(s)\|_{V}^{2}\left[C\left(\alpha_{1}\right)+16\left(\mathcal{P}^{2}+\alpha_{1}\right)\left(1-\mathrm{e}^{-K_{1}(t-s)}\right)\right]
$$

Hence, using (3.23), we obtain

$$
y(t)<M-\int_{0}^{t}(M-y(s)) a(s, t) \mathrm{d} s .
$$

As $y$ is a continuous solution of (3.9) and as $0 \leq y(0)=\left\|\mathbf{u}_{0}\right\|_{V_{2}}^{2}<M$ owing to (3.23), there is an interval of time on which $y(t)<M$. Let us prove, by contradiction, that this interval is $\mathbb{R}^{+}$. Indeed, if this were not true, there would exist $t^{*}>0$ such that:

$$
\forall t<t^{*}, 0 \leq y(t)<M \text { and } y\left(t^{*}\right)=M
$$

therefore $y \leq M$ on $\left[0, t^{*}\right]$ whereas the relation (3.25) gives: $y\left(t^{*}\right)<M$, thus contradicting the equality in (3.26).

We conclude this section in proving uniqueness of a global solution of problem $(2.20,2.4)$, if it exists. Let us set

$$
\mathbf{K}(\mathbf{v})=-\operatorname{div}\left(|A(\mathbf{v})|^{2} A(\mathbf{v})\right)
$$

and let us show that the operator $\mathbf{K}$ is monotonous.

Lemma 3.5. Let $\mathbf{K}$ be defined by (3.27). For any $\mathbf{v}_{1}$ and any $\mathbf{v}_{2}$ in $V_{2}$,

$$
\left(K\left(\mathbf{v}_{1}\right)-K\left(\mathbf{v}_{2}\right), \mathbf{v}_{1}-\mathbf{v}_{2}\right) \geq 0 .
$$

Proof. Let us recall that, owing to Lemma 2.4,

$$
(\mathbf{K}(\mathbf{v}), \mathbf{w})=\frac{1}{2}\left(|A(\mathbf{v})|^{2} A(\mathbf{v}), A(\mathbf{w})\right) .
$$

Hence

$$
2\left(\mathbf{K}\left(\mathbf{v}_{1}\right)-\mathbf{K}\left(\mathbf{v}_{2}\right), \mathbf{v}_{1}-\mathbf{v}_{2}\right) \geq\left\|\left|A\left(\mathbf{v}_{1}\right)\right|\right\|_{L^{4}(\Omega}^{4}+\left\|\left|A\left(\mathbf{v}_{2}\right)\right|\right\|_{L^{4}(\Omega}^{4}-\frac{1}{2}\left\|\left|A\left(\mathbf{v}_{1}\right)\right|^{2}+\left|A\left(\mathbf{v}_{2}\right)\right|^{2}\right\|_{L^{2}(\Omega)}^{2}
$$


If we set

$$
w_{1}=\left|A\left(\mathbf{v}_{1}\right)\right|^{2} \text { and } w_{2}=\left|A\left(\mathbf{v}_{2}\right)\right|^{2},
$$

the previous inequality can be written

$$
2\left(\mathbf{K}\left(\mathbf{v}_{1}\right)-\mathbf{K}\left(\mathbf{v}_{2}\right), \mathbf{v}_{1}-\mathbf{v}_{2}\right) \geq\left\|w_{1}\right\|_{L^{2}(\Omega)}^{2}+\left\|w_{2}\right\|_{L^{2}(\Omega)}^{2}-\frac{1}{2}\left\|w_{1}+w_{2}\right\|_{L^{2}(\Omega)}^{2},
$$

which implies (3.28).

The following lemma refers to any pair of solutions of (2.20).

Lemma 3.6. Let $\mathbf{u}_{1}$ and $\mathbf{u}_{2}$ be two solutions of (2.20). Their difference $\mathbf{u}=\mathbf{u}_{1}-\mathbf{u}_{2}$ satisfies the equality:

$$
\begin{aligned}
\frac{1}{2} \frac{\mathrm{d}}{\mathrm{d} t}\|\mathbf{u}(t)\|_{V}^{2}+\nu|\mathbf{u}(t)|_{H^{1}(\Omega)}^{2}+ & b\left(\mathbf{u}(t) ; \mathbf{u}_{2}(t)-2\left(\alpha_{1}+\alpha_{2}\right) \Delta \mathbf{u}_{2}(t), \mathbf{u}(t)\right) \\
& +b\left(\mathbf{u}(t) ; \Delta \mathbf{u}(t),\left(2 \alpha_{1}+\alpha_{2}\right) \mathbf{u}_{2}(t)+\left(\alpha_{1}+\alpha_{2}\right) \mathbf{u}_{1}(t)\right) \\
+ & b\left(2\left(\alpha_{1}+\alpha_{2}\right) \mathbf{u}_{1}(t)-\alpha_{1} \mathbf{u}_{2}(t) ; \Delta \mathbf{u}(t), \mathbf{u}(t)\right)+\beta\left(\mathbf{K}\left(\mathbf{u}_{1}(t)\right)-\mathbf{k}\left(\mathbf{u}_{2}(t)\right), \mathbf{u}(t)\right)=0 .
\end{aligned}
$$

Proof. The proof derives from (2.20) and the following relation:

$$
((\operatorname{curl} \mathbf{u}) \times \mathbf{v}, \mathbf{w})=b(\mathbf{v} ; \mathbf{u} ; \mathbf{w})-b(\mathbf{w} ; \mathbf{u}, \mathbf{v}) .
$$

Theorem 3.7. Problem (2.20, 2.4) has at most one solution in $L^{\infty}\left(0, T ; V_{2}\right)$ for any $T>0$.

Proof. Suppose that problem $(2.20,2.4)$ has two solutions $\mathbf{u}_{1}$ and $\mathbf{u}_{2}$ in $L^{\infty}\left(0, T ; V_{2}\right)$ for any $T>0$ and set $\mathbf{u}=\mathbf{u}_{1}-\mathbf{u}_{2}$. Considering that $\mathbf{u}_{2}$ belongs to $L^{\infty}\left(0, T ; V_{2}\right)$, the estimates derived in the proof of Theorem 3.3 yield

$$
\left|b\left(\mathbf{u}(t) ; \mathbf{u}_{2}(t)-2\left(\alpha_{1}+\alpha_{2}\right) \Delta \mathbf{u}_{2}(t), \mathbf{u}(t)\right)\right| \leq c_{1}(T)|\mathbf{u}(t)|_{H^{1}(\Omega)}^{2},
$$

where $c_{1}(T)$ and the subsequent constants $c_{2}(T)$ and $c_{3}(T)$ depend on $\alpha_{1}, \alpha_{2}, \mathcal{P}, C_{1}, C_{2}, C\left(\alpha_{1}\right),\left\|\mathbf{u}_{1}\right\|_{L \infty\left(0, T ; V_{2}\right)}$, and $\left\|\mathbf{u}_{2}\right\|_{L^{\infty}\left(0, T ; V_{2}\right)}$ and are bounded since all these quantities are bounded. Next, as in the proof of Lemma 3.1, Green's formula gives

$$
b(\mathbf{u} ; \Delta \mathbf{u}, \mathbf{v})=\sum_{i=1}^{3} b\left(\frac{\partial \mathbf{u}}{\partial x_{i}} ; \mathbf{v}, \frac{\partial \mathbf{u}}{\partial x_{i}}\right)+\sum_{i=1}^{3} b\left(\mathbf{u} ; \frac{\partial \mathbf{v}}{\partial x_{i}}, \frac{\partial \mathbf{u}}{\partial x_{i}}\right) .
$$

Hence

$$
\left|b\left(\mathbf{u}(t) ; \Delta \mathbf{u}(t),\left(2 \alpha_{1}+\alpha_{2}\right) \mathbf{u}_{2}(t)+\left(\alpha_{1}+\alpha_{2}\right) \mathbf{u}_{1}(t)\right)\right| \leq c_{2}(T)|\mathbf{u}(t)|_{H^{1}(\Omega)}^{2} .
$$

Similarly, we have

$$
\left|b\left(\alpha_{1} \mathbf{u}_{2}(t)-2\left(\alpha_{1}+\alpha_{2}\right) \mathbf{u}_{1}(t) ; \Delta \mathbf{u}(t), \mathbf{u}(t)\right)\right| \leq c_{3}(T)|\mathbf{u}(t)|_{H^{1}(\Omega)}^{2} .
$$

Substituting these inequalities into (3.29) and using $(3.4,3.28)$, we obtain

$$
\frac{\mathrm{d}}{\mathrm{d} t}\|\mathbf{u}(t)\|_{V}^{2} \leq 2 \frac{c_{1}(T)+c_{2}(T)+c_{3}(T)}{\alpha_{1}}\|\mathbf{u}(t)\|_{V}^{2} .
$$

Then Gronwall's inequality and the fact that $\mathbf{u}(0)=\mathbf{0}$ imply that $\mathbf{u}(t)=\mathbf{0}$ for all $t$ in $[0, T]$. 


\section{Existence of SOLUtion}

In this section, we assume that the boundary $\Gamma$ of $\Omega$ is of class $C^{2,1}$ and $\mathbf{f}$ belongs to $L^{2}\left(\mathbb{R}^{+} ; H(\mathbf{c u r l} ; \Omega)\right) \bigcap$ $L^{\infty}\left(\mathbb{R}^{+} ; L^{2}(\Omega)^{3}\right)$.

The solution of problem $(2.20,2.4)$ is constructed by means of Galerkin's discretization. As the imbedding $V_{2} \subset V$ is compact, there exists a sequence of eigenfunctions $\left\{\mathbf{w}_{j}\right\}$ in $V_{2}$ corresponding to a sequence of eigenvalues $\left\{\lambda_{j}\right\}$ such that:

$$
\left(\mathbf{w}_{j}, \mathbf{v}\right)_{V_{2}}=\lambda_{j}\left(\mathbf{w}_{j}, \mathbf{v}\right)_{V}, \forall \mathbf{v} \in V_{2}
$$

with

$$
0<\lambda_{1}<\ldots<\lambda_{k}<\ldots \rightarrow+\infty
$$

The functions $\mathbf{w}_{j}$ form an orthonormal basis in $V$ and an orthogonal basis in $V_{2}$. Following the approach of Cioranescu and Ouazar in [7], this set of functions will be used as a special basis for the Galerkin-Faedo method. The two following lemmas establish properties of the eigenfunctions $\mathbf{w}_{j}$.

Lemma 4.1. Under the assumptions of Lemma 2.1, the eigenfunctions $\mathbf{w}_{j}$ of (4.1) are such that $\operatorname{curl}\left(\Delta \mathbf{w}_{j}\right)$ belongs to $H^{1}(\Omega)^{3}$.

Proof. This result is established in the proof of a lemma of [5].

Lemma 4.2. The eigenfunctions $\mathbf{w}_{j}$ of (4.1) satisfy, for $j \geq 1$,

$$
\forall \mathbf{g} \in H(\operatorname{curl} ; \Omega), \quad\left(\operatorname{curl} \mathbf{g}, \operatorname{curl}\left(\mathbf{w}_{j}-\alpha_{1} \Delta \mathbf{w}_{j}\right)\right)=\lambda_{j}\left(\mathbf{g}, \mathbf{w}_{j}\right) .
$$

Proof. Let $\mathbf{g}$ belong to $H(\operatorname{curl} ; \Omega)$ and $\mathbf{v}$ be the solution in $V$ of the Stokes problem:

$$
\mathbf{v}-\alpha_{1} \Delta \mathbf{v}+\nabla p=\mathbf{g}
$$

On the one hand, Lemma 2.1 implies that $\mathbf{v}$ belongs to $V_{2}$. On the other hand, the equality (4.1) yields

$$
\forall \mathbf{v} \in V_{2},\left(\operatorname{curl}\left(\mathbf{v}-\alpha_{1} \Delta \mathbf{v}\right), \operatorname{curl}\left(\mathbf{w}_{j}-\alpha_{1} \Delta \mathbf{w}_{j}\right)\right)=\lambda_{j}\left(\mathbf{v}-\alpha_{1} \Delta \mathbf{v}, \mathbf{w}_{j}\right)
$$

Then the result derives from $(4.2,4.3)$.

For any positive integer $m$, we denote by $V_{m}$ the vector space spanned by the first $m$ eigenfunctions $\left\{\mathbf{w}_{j}\right\}_{j=1}^{m}$ and by $P_{m}$ the orthogonal projection operator on $V_{m}$ for the scalar product in $V_{2}$. We define an approximate solution of problem $(2.20,2.4)$ by: Find

$$
\mathbf{u}_{m}(t)=\sum_{j=1}^{m} c_{j, m}(t) \mathbf{w}_{j}
$$


solution for $1 \leq j \leq m$, of

$$
\begin{aligned}
& \left(\mathbf{u}_{m}^{\prime}(t), \mathbf{w}_{j}\right)_{V}+\nu\left(\nabla \mathbf{u}_{m}(t), \nabla \mathbf{w}_{j}\right)+\left(\operatorname{curl}\left(\mathbf{u}_{m}(t)-\left(2 \alpha_{1}+\alpha_{2}\right) \Delta \mathbf{u}_{m}(t)\right) \times \mathbf{u}_{m}(t), \mathbf{w}_{j}\right) \\
& \quad+\left(\alpha_{1}+\alpha_{2}\right)\left\{b\left(\mathbf{u}_{m}(t) ; \Delta \mathbf{w}_{j}, \mathbf{u}_{m}(t)\right)+2 b\left(\mathbf{u}_{m}(t) ; \Delta \mathbf{u}_{m}(t), \mathbf{w}_{j}\right)\right\}+\frac{\beta}{2}\left(\left|A\left(\mathbf{u}_{m}(t)\right)\right|^{2} A\left(\mathbf{u}_{m}(t)\right), A\left(\mathbf{w}_{j}\right)\right)=\left(\mathbf{f}(t), \mathbf{w}_{j}\right)
\end{aligned}
$$

$$
\mathbf{u}_{m}(0)=P_{m}\left(\mathbf{u}_{0}\right)
$$

Classical results on ODE (cf. [8]) insure that such a system has a solution $\mathbf{u}_{m}$, unique and continuous on $\left[0, T_{m}^{*}\right]$ with $\mathbf{u}_{m}^{\prime}$ in $L^{\infty}\left(0, T_{m}^{*}\right)$, for some number $T_{m}^{*}>0$. We propose to prove that $\mathbf{u}_{m}(t)$ satisfies the a priori estimates of Section 3.

On multiplying both sides of (4.4) by $c_{j, m}(t)$ and summing with respect to $j$, we obtain on $\left[0, T_{m}^{*}\right]$ the equality (compare with (3.3)):

$$
\frac{1}{2} \frac{\mathrm{d}}{\mathrm{d} t}\left\|\mathbf{u}_{m}(t)\right\|_{V}^{2}+\nu\left|\mathbf{u}_{m}(t)\right|_{H^{1}(\Omega)}^{2}+\frac{\beta}{2}\left\|\left|A\left(\mathbf{u}_{m}(t)\right)\right|\right\|_{L^{4}(\Omega)}^{4}+3\left(\alpha_{1}+\alpha_{2}\right) b\left(\mathbf{u}_{m}(t) ; \Delta \mathbf{u}_{m}(t), \mathbf{u}_{m}(t)\right)=\left(\mathbf{f}(t), \mathbf{u}_{m}(t)\right) .
$$

Then, the proof of Lemma 3.1 carries over to $\mathbf{u}_{m}$ without modification and yields the following result.

Lemma 4.3. The solution $\mathbf{u}_{m}$ of problem $(4.4,4.5)$ satisfies the inequalities for all $t$ in $\left[0, T_{m}^{*}\right]$ :

$$
\begin{aligned}
\left\|\mathbf{u}_{m}(t)\right\|_{V}^{2} \leq & \mathrm{e}^{-K_{1} t}\left\|\mathbf{u}_{m}(0)\right\|_{V}^{2}+\frac{\mathcal{P}^{2}}{\nu} \int_{0}^{t} \mathrm{e}^{-K_{1}(t-s)}\|\mathbf{f}(s)\|_{L^{2}(\Omega)}^{2} \mathrm{~d} s \\
& -K_{2} \int_{0}^{t} \mathrm{e}^{-K_{1}(t-s)}\left(\frac{K_{1}}{K_{2}}-\left\|\mathbf{u}_{m}(s)\right\|_{V_{2}}\right)\left\|\mathbf{u}_{m}(s)\right\|_{V}^{2} \mathrm{~d} s \\
\int_{0}^{t}\left\|\left|A\left(\mathbf{u}_{m}(s)\right)\right|\right\|_{L^{4}(\Omega)}^{4} \mathrm{~d} s \leq & \frac{1}{\beta}\left(\left\|\mathbf{u}_{m}(0)\right\|_{V}^{2}+\frac{\mathcal{P}^{2}}{\nu} \int_{0}^{t}\|\mathbf{f}(s)\|_{L^{2}(\Omega)}^{2} \mathrm{~d} s\right) \\
& \left.-\frac{K_{2}}{\beta} \int_{0}^{t}\left(\frac{K_{1}}{K_{2}}-\left\|\mathbf{u}_{m}(s)\right\|_{V_{2}}\right)\left\|\mathbf{u}_{m}(s)\right\|_{V}^{2} \mathrm{~d} s\right)
\end{aligned}
$$

where $K_{1}$ and $K_{2}$ are defined as in Lemma 3.1.

Owing to the special basis, we can also derive from equation (4.4) an estimate for $\operatorname{curl}\left(\mathbf{u}_{m}(t)-\alpha_{1} \Delta \mathbf{u}_{m}(t)\right)$. We define, first, the vector valued function $\mathbf{F}(\mathbf{v})$ for all $\mathbf{v}$ in $V_{2}$ :

$$
\begin{aligned}
\mathbf{F}(\mathbf{v})= & -\nu \Delta \mathbf{v}+\operatorname{curl}\left(\mathbf{v}-\left(2 \alpha_{1}+\alpha_{2}\right) \Delta \mathbf{v}\right) \times \mathbf{v} \\
& +\left(\alpha_{1}+\alpha_{2}\right)(-\Delta(\mathbf{v} \cdot \nabla \mathbf{v})+2 \mathbf{v} \cdot \nabla(\Delta \mathbf{v}))-\beta \operatorname{div}\left(|A(\mathbf{v})|^{2} A(\mathbf{v})\right) .
\end{aligned}
$$

Using the definition of $\mathbf{F}$, we obtain

$$
\left(\mathbf{u}_{m}^{\prime}(t), \mathbf{w}_{j}\right)_{V}+\left(\mathbf{F}\left(\mathbf{u}_{m}(t)\right)-\mathbf{f}(t), \mathbf{w}_{j}\right)=0 .
$$

Owing to Lemma $4.1, \mathbf{F}\left(\mathbf{u}_{m}(t)\right)$ belongs to $H(\mathbf{c u r l} ; \Omega)$. Then, multiplying the previous equality by $c_{j, m}(t) \lambda_{j}$ and setting $\mathbf{z}_{m}=\operatorname{curl}\left(\mathbf{u}_{m}-\alpha_{1} \Delta \mathbf{u}_{m}\right)$, Lemma 4.2 yields

$$
\frac{1}{2} \frac{\mathrm{d}}{\mathrm{d} t}\left\|\mathbf{u}_{m}(t)\right\|_{V_{2}}^{2}+\left(\operatorname{curl} \mathbf{F}\left(\mathbf{u}_{m}(t)\right), \mathbf{z}_{m}(t)\right)=\left(\operatorname{curl} \mathbf{f}(t), \mathbf{z}_{m}(t)\right) .
$$

The next theorem establishes the analogue of Theorem 3.3. 
Theorem 4.4. Suppose that $\mathbf{f}$ belongs to $L^{2}\left(\mathbb{R}^{+} ; H(\operatorname{curl} ; \Omega)\right) \bigcap L^{\infty}\left(\mathbb{R}^{+} ; L^{2}(\Omega)^{3}\right)$ and $\Gamma$ is of class $C^{2,1}$. Then $y(t)=\left\|\mathbf{u}_{m}(t)\right\|_{V_{2}}^{2}$ satisfies the differential inequality in $\left[0, T_{m}^{*}\right]$ :

$$
\begin{aligned}
y^{\prime}(t) \leq & \frac{4 \nu}{\alpha_{1}^{2}}\left(\mathrm{e}^{-K_{1} t}\left\|\mathbf{u}_{m}(0)\right\|_{V}^{2}+\frac{\mathcal{P}^{2}}{\nu} \int_{0}^{t} \mathrm{e}^{-K_{1}(t-s)}\|\mathbf{f}(s)\|_{L^{2}(\Omega)}^{2} \mathrm{~d} s\right) \\
& +\frac{2 \alpha_{1}}{\nu}\|\mathbf{c u r l} \mathbf{f}(t)\|_{L^{2}(\Omega)}^{2}-C\left(\alpha_{1}, \alpha_{2}, \beta\right)\left(\frac{\nu}{2 \alpha_{1} C\left(\alpha_{1}, \alpha_{2}, \beta\right)}-y(t)\right) y(t) \\
& +\frac{\beta C\left(\alpha_{1}\right)}{2 \alpha_{1}^{2}}\left\|\left|A\left(\mathbf{u}_{m}(t)\right)\right|\right\|_{L^{4}(\Omega)}^{4}-\frac{4 \nu}{\alpha_{1}^{2}} K_{2} \int_{0}^{t} \mathrm{e}^{-K_{1}(t-s)}\left(\frac{K_{1}}{K_{2}}-\sqrt{y(s)}\right)\left\|\mathbf{u}_{m}(s)\right\|_{V}^{2} \mathrm{~d} s,
\end{aligned}
$$

where $C\left(\alpha_{1}, \alpha_{2}, \beta\right)$ is defined by (3.10) and $K_{1}$ and $K_{2}$ are defined as in Lemma 3.1.

Proof. Thanks to $(2.27-2.29,2.22)$, from (4.10) we derive after suppressing the variable $t$, in order to simplify the notation, and after setting $\boldsymbol{\omega}_{m}=\operatorname{curl} \mathbf{u}_{m}$,

$$
\begin{aligned}
& \frac{1}{2} \frac{\mathrm{d}}{\mathrm{d} t}\left\|\mathbf{u}_{m}\right\|_{V_{2}}^{2}+\frac{\nu}{\alpha_{1}}\left\|\mathbf{u}_{m}\right\|_{V_{2}}^{2}-b\left(\mathbf{z}_{m} ; \mathbf{u}_{m}, \mathbf{z}_{m}\right)+\left(\alpha_{1}+\alpha_{2}\right)\left\{-b\left(\Delta \mathbf{u}_{m} ; \boldsymbol{\omega}_{m}, \mathbf{z}_{m}\right)\right. \\
&+b\left(\boldsymbol{\omega}_{m} ; \Delta \mathbf{u}_{m}, \mathbf{z}_{m}\right)+2 \sum_{k=1}^{3}\left[b\left(\frac{\partial \boldsymbol{\omega}_{m}}{\partial x_{k}} ; \frac{\partial \mathbf{u}_{m}}{\partial x_{k}}, \mathbf{z}_{m}\right)-b\left(\frac{\partial \mathbf{u}_{m}}{\partial x_{k}} ; \frac{\partial \boldsymbol{\omega}_{m}}{\partial x_{k}}, \mathbf{z}_{m}\right)\right. \\
&\left.\left.+\left(\nabla u_{k m} \times \nabla \Delta u_{k m}, \mathbf{z}_{m}\right)\right]\right\}-\beta\left\{2\left(\nabla\left(\left|A\left(\mathbf{u}_{m}\right)\right|^{2}\right) . \nabla \boldsymbol{\omega}_{m}, \mathbf{z}_{m}\right)+\left(\mathbf{B}\left(\mathbf{u}_{m}\right), \mathbf{z}_{m}\right)\right\} \\
&+\frac{\beta}{\alpha_{1}}\left\|\left|A\left(\mathbf{u}_{m}\right)\right| \mathbf{z}_{m}\right\|_{L^{2}(\Omega)}^{2}=\left(\operatorname{curl}\left(\mathbf{f}+\frac{\nu}{\alpha_{1}} \mathbf{u}_{m}\right), \mathbf{z}_{m}\right)+\frac{\beta}{\alpha_{1}}\left(\left|A\left(\mathbf{u}_{m}\right)\right|^{2} \mathbf{c u r l} \mathbf{u}_{m}, \mathbf{z}_{m}\right) .
\end{aligned}
$$

This is exactly the same situation as in Theorem 3.3 and the same proof gives (4.11).

Consider a solution of (4.11) with initial value

$$
y(0)=\left\|\mathbf{u}_{m}(0)\right\|_{V_{2}}^{2}=\left\|P_{m}\left(\mathbf{u}_{0}\right)\right\|_{V_{2}}^{2}
$$

The convergence properties of $P_{m}$ imply that, if $\mathbf{u}_{0}$ and $\mathbf{f}$ satisfy (3.23), then for all sufficiently large $m, \mathbf{u}_{m}(0)$ and $\mathbf{f}$ will satisfy the analogue of $(3.23)$ :

$$
\begin{aligned}
\left\|\mathbf{u}_{m}(0)\right\|_{V_{2}}^{2}+\frac{8 \nu+C\left(\alpha_{1}\right) K_{1}}{2 \alpha_{1}^{2} K_{1}}\left(\left\|\mathbf{u}_{m}(0)\right\|_{V}^{2}\right. & \left.+\frac{\mathcal{P}^{2}}{\nu} \int_{0}^{\infty}\|\mathbf{f}(t)\|_{L^{2}(\Omega)}^{2} \mathrm{~d} t\right)+\frac{2 \alpha_{1}}{\nu} \int_{0}^{\infty}\|\mathbf{c u r l} \mathbf{f}(t)\|_{L^{2}(\Omega)}^{2} \mathrm{~d} t \\
& <\min \left(\frac{\nu}{2 \alpha_{1} C\left(\alpha_{1}, \alpha_{2}, \beta\right)}, \frac{K_{1}^{2}}{K_{2}^{2}}\right) .
\end{aligned}
$$

Hence the conclusion of Lemma 3.4 implies that $T_{m}^{*}=\infty$ and that $\mathbf{u}_{m}(t)$ is uniformly bounded in $V_{2}$ with respect to time:

$$
\forall t \geq 0,\left\|\mathbf{u}_{m}(t)\right\|_{V_{2}} \leq \min \left(\sqrt{\frac{\nu}{2 \alpha_{1} C\left(\alpha_{1}, \alpha_{2}, \beta\right)}}, \frac{K_{1}}{K_{2}}\right)
$$

Thus, the equivalence of norms of Lemma $2.1,(4.7,4.14)$ imply that the sequence $\left\{\mathbf{u}_{m}\right\}_{m \geq 1}$ is bounded with respect to $m$ in $L^{\infty}\left(\mathbb{R}^{+} ; H^{3}(\Omega)^{3}\right) \bigcap L^{2}\left(\mathbb{R}^{+} ; H^{1}(\Omega)^{3}\right)$.

The next lemma gives a bound for $\mathbf{u}_{m}^{\prime}(t)$. 
Lemma 4.5. Let $\mathbf{f}$ belong to $L^{2}\left(\mathbb{R}^{+} ; L^{2}(\Omega)^{3}\right)$ and $\mathbf{u}_{0}$ belong to $V_{2}$. Suppose that the sequence $\left\{\mathbf{u}_{m}\right\}_{m \geq 1}$ is bounded with respect to $m$ in $L^{\infty}\left(\mathbb{R}^{+} ; H^{3}(\Omega)^{3}\right) \cap L^{2}\left(\mathbb{R}^{+} ; H^{1}(\Omega)^{3}\right)$. Then $\left\{\mathbf{u}_{m}^{\prime}\right\}_{m \geq 1}$ is bounded with respect to $m$ in $L^{2}\left(\mathbb{R}^{+} ; V\right)$.

Proof. Let us multiply both sides of (4.4) by $c_{j m}^{\prime}(t)$, sum over $j$ and use (3.30), this gives:

$$
\begin{aligned}
\left\|\mathbf{u}_{m}^{\prime}(t)\right\|_{V}^{2}= & \nu\left(\Delta \mathbf{u}_{m}(t), \mathbf{u}_{m}^{\prime}(t)\right)-b\left(\mathbf{u}_{m}(t) ; \mathbf{u}_{m}(t), \mathbf{u}_{m}^{\prime}(t)\right)-\alpha_{2} b\left(\mathbf{u}_{m}(t) ; \Delta \mathbf{u}_{m}(t), \mathbf{u}_{m}^{\prime}(t)\right) \\
& -\left(2 \alpha_{1}+\alpha_{2}\right) b\left(\mathbf{u}_{m}^{\prime}(t) ; \Delta \mathbf{u}_{m}(t), \mathbf{u}_{m}(t)\right)-\left(\alpha_{1}+\alpha_{2}\right) b\left(\mathbf{u}_{m}(t) ; \Delta \mathbf{u}_{m}^{\prime}(t), \mathbf{u}_{m}(t)\right) \\
& -\frac{\beta}{2}\left(\left|A\left(\mathbf{u}_{m}(t)\right)\right|^{2} A\left(\mathbf{u}_{m}(t)\right), A\left(\mathbf{u}_{m}^{\prime}(t)\right)\right)+\left(\mathbf{f}(t), \mathbf{u}_{m}^{\prime}(t)\right) .
\end{aligned}
$$

The arguments of Section 3 show that: $b\left(\mathbf{u}_{m}(t) ; \Delta \mathbf{u}_{m}^{\prime}(t), \mathbf{u}_{m}(t)\right), b\left(\mathbf{u}_{m}(t) ; \mathbf{u}_{m}(t), \mathbf{u}_{m}^{\prime}(t)\right), b\left(\mathbf{u}_{m}^{\prime}(t) ; \Delta \mathbf{u}_{m}(t), \mathbf{u}_{m}(t)\right)$ and $b\left(\mathbf{u}_{m}(t) ; \Delta \mathbf{u}_{m}(t), \mathbf{u}_{m}^{\prime}(t)\right)$ are all bounded by an expression of the form

$$
C\left|\mathbf{u}_{m}(t)\right|_{H^{1}(\Omega)}\left\|\mathbf{u}_{m}(t)\right\|_{H^{3}(\Omega)}\left|\mathbf{u}_{m}^{\prime}(t)\right|_{H^{1}(\Omega)},
$$

where the constant $C$ is independent of $m$ and $t$. Moreover,

$$
\left(\left|A\left(\mathbf{u}_{m}(t)\right)\right|^{2} A\left(\mathbf{u}_{m}(t)\right), A\left(\mathbf{u}_{m}^{\prime}(t)\right)\right)=\frac{1}{4} \frac{\mathrm{d}}{\mathrm{d} t}\left(\left\|\left|A\left(\mathbf{u}_{m}(t)\right)\right|\right\|_{L^{4}(\Omega)}^{4}\right) .
$$

Hence, we obtain

$$
\begin{aligned}
\left\|\mathbf{u}_{m}^{\prime}(t)\right\|_{V}^{2}+\frac{\beta}{8} \frac{\mathrm{d}}{\mathrm{d} t}\left(\left\|\left|A\left(\mathbf{u}_{m}(t)\right)\right|\right\|_{L^{4}(\Omega)}^{4}\right) \leq & \nu\left|\mathbf{u}_{m}(t)\right|_{H^{1}(\Omega)}\left|\mathbf{u}_{m}^{\prime}(t)\right|_{H^{1}(\Omega)}+\|\mathbf{f}(t)\|_{L^{2}(\Omega)}\left\|\mathbf{u}_{m}^{\prime}(t)\right\|_{L^{2}(\Omega)} \\
& +k\left\|\mathbf{u}_{m}(t)\right\|_{H^{3}(\Omega)}\left|\mathbf{u}_{m}(t)\right|_{H^{1}(\Omega)}\left|\mathbf{u}_{m}^{\prime}(t)\right|_{H^{1}(\Omega)}
\end{aligned}
$$

where the constant $\mathrm{k}$ is independent of $m$ and $t$. Using (3.4) and the identity $a b \leq a^{2}+(1 / 4) b^{2}$, integrating over $\mathbb{R}^{+}$yields

$$
\begin{aligned}
\left\|\mathbf{u}_{m}^{\prime}\right\|_{L^{2}\left(\mathbb{R}^{+} ; V\right)}^{2} \leq & \frac{2}{\alpha_{1}}\left(\nu+k\left\|\mathbf{u}_{m}\right\|\right. \\
& L^{\infty}\left(\mathbb{R}^{+} ; H^{3}(\Omega)^{3}\right)^{2}\left\|\mathbf{u}_{m}\right\|_{L^{2}}^{2}\left(\mathbb{R}^{+} ; H^{1}(\Omega)^{3}\right) \\
& +2\|\mathbf{f}\|_{L^{2}\left(\mathbb{R}^{+} ; L^{2}(\Omega)^{3}\right)}^{2}+\frac{\beta}{4}\left\|\left|A\left(\mathbf{u}_{m}(0)\right)\right|\right\|_{L^{4}(\Omega)}^{4} .
\end{aligned}
$$

Since we have $\left\|\left|A\left(\mathbf{u}_{m}(0)\right)\right|\right\|_{L^{4}(\Omega)}^{4} \leq 16 C_{1}^{2} / \alpha_{1}\left\|\mathbf{u}_{m}\right\|_{L^{\infty}\left(\mathbb{R}^{+} ; H^{3}(\Omega)^{3}\right)}^{2}\left\|\mathbf{u}_{0}\right\|_{V}^{2}$, the sequence $\left\{\mathbf{u}_{m}^{\prime}\right\}_{m \geq 1}$ is bounded in $L^{2}\left(\mathbb{R}^{+} ; V\right)$.

The next theorem summarizes the above bounds.

Theorem 4.6. Let $\Omega$ be a bounded, simply-connected open set of $\mathbb{R}^{3}$ with a boundary $\Gamma$ of class $C^{2,1}$. Let the right-hand side $\mathbf{f}$ be given in $\left.L^{2}\left(\mathbb{R}^{+} ; H(\mathbf{c u r l} ; \Omega)\right) \cap L^{\infty}\left(\mathbb{R}^{+} ; L^{2}(\Omega)^{3}\right)\right)$ and the initial velocity $\mathbf{u}_{0}$ be given in $V_{2}$, small enough so that they satisfy

$$
\begin{aligned}
\left\|\mathbf{u}_{0}\right\|_{V_{2}}^{2}+\frac{8 \nu+C\left(\alpha_{1}\right) K_{1}}{2 \alpha_{1}^{2} K_{1}}\left(\left\|\mathbf{u}_{0}\right\|_{V}^{2}\right. & \left.+\frac{\mathcal{P}^{2}}{\nu} \int_{0}^{\infty}\|\mathbf{f}(t)\|_{L^{2}(\Omega)}^{2} \mathrm{~d} t\right)+\frac{2 \alpha_{1}}{\nu} \int_{0}^{\infty}\|\operatorname{curl} \mathbf{f}(t)\|_{L^{2}(\Omega)}^{2} \mathrm{~d} t \\
& <\min \left(\frac{\nu}{2 \alpha_{1} C\left(\alpha_{1}, \alpha_{2}, \beta\right)}, \frac{K_{1}^{2}}{K_{2}^{2}}\right)
\end{aligned}
$$

with $C\left(\alpha_{1}, \alpha_{2}, \beta\right)$ defined by (3.10) and $K_{1}$ and $K_{2}$ defined as in Lemma 3.1. Then for all sufficiently large $m$, the unique solution $\mathbf{u}_{m}$ of the Galerkin system of equations $(4.4,4.5)$ exists for all time $t \geq 0$ and satisfies the 
upper bounds:

$$
\begin{array}{r}
\left\|\mathbf{u}_{m}\right\|_{L^{\infty}\left(\mathbb{R}^{+} ; V_{2}\right)} \leq \min \left(\sqrt{\frac{\nu}{2 \alpha_{1} C\left(\alpha_{1}, \alpha_{2}, \beta\right)}}, \frac{K_{1}}{K_{2}}\right) \\
\left\|\mathbf{u}_{m}\right\|_{L^{2}\left(\mathbb{R}^{+} ; H^{1}(\Omega)^{3}\right)} \leq k_{1} \quad \text { and } \quad\left\|\mathbf{u}_{m}^{\prime}\right\|_{L^{2}\left(\mathbb{R}^{+} ; V\right)} \leq k_{2},
\end{array}
$$

where $k_{1}$ and $k_{2}$ are constants independent of $m$.

It remains to pass to the limit with respect to $m$. It follows from (4.17) that there exists a function $\mathbf{u}$ and a subsequence of $\left\{\mathbf{u}_{m}\right\}$, still denoted $\left\{\mathbf{u}_{m}\right\}$, such that

$$
\begin{aligned}
& \lim _{m \rightarrow \infty} \mathbf{u}_{m}=\mathbf{u} \text { weak }^{*} \text { in } L^{\infty}\left(\mathbb{R}^{+} ; V_{2}\right), \\
& \lim _{m \rightarrow \infty} \mathbf{u}_{m}^{\prime}=\mathbf{u}^{\prime} \text { weak in } L^{2}\left(\mathbb{R}^{+} ; V\right) .
\end{aligned}
$$

On one hand, this implies that

$$
\|\mathbf{u}\|_{L^{\infty}\left(\mathbb{R}^{+} ; V_{2}\right)} \leq \min \left(\sqrt{\frac{\nu}{2 \alpha_{1} C\left(\alpha_{1}, \alpha_{2}, \beta\right)}}, \frac{K_{1}}{K_{2}}\right) \text { and } \mathbf{u}(0)=\mathbf{u}_{0} .
$$

On the other hand, for any $T>0,\left\{\mathbf{u}_{m}\right\}_{m \geq 0}$ is bounded in the space

$$
W=\left\{\mathbf{v} \in L^{2}\left(0, T ; H^{3}(\Omega)^{3}\right), \mathbf{v}^{\prime} \in L^{2}\left(0, T ; H^{1}(\Omega)^{3}\right)\right\}
$$

According to [11], the imbedding of $W$ into $L^{2}\left(0, T ; H^{2}(\Omega)^{3}\right)$ is compact; thus

$$
\lim _{m \rightarrow \infty} \mathbf{u}_{m}=\mathbf{u} \text { strongly in } L^{2}\left(0, T ; H^{2}(\Omega)^{3}\right) .
$$

The only problem is to pass to the limit in the term $\mathbf{K}\left(\mathbf{u}_{m}\right)$. We have

$$
\lim _{m \rightarrow \infty} \mathbf{K}\left(\mathbf{u}_{m}\right)=\boldsymbol{\Psi} \text { weak in } L^{2}\left(0, T ; L^{2}(\Omega)^{3}\right) .
$$

It remains to prove $\boldsymbol{\Psi}=\mathbf{K}(\mathbf{u})$. First, we have

$$
\forall(\mathbf{u}, \mathbf{w}) \in\left(H^{3}(\Omega)^{3}\right)^{2}, \forall \mathbf{v} \in H_{0}^{1}(\Omega)^{3}, \lim _{\varepsilon \rightarrow 0}(\mathbf{K}(\mathbf{u}+\varepsilon \mathbf{w}), \mathbf{v})=(\mathbf{K}(\mathbf{u}), \mathbf{v}) .
$$

Next, for any w in $L^{\infty}\left(0, T ; H^{3}(\Omega)^{3} \cap H_{0}^{1}(\Omega)^{3}\right)$ and any $\varepsilon>0,(3.28)$ implies

$$
\int_{0}^{T}\left(\mathbf{K}\left(\mathbf{u}_{m}(t)\right)-\mathbf{K}(\mathbf{u}(t)-\varepsilon \mathbf{w}(t)), \mathbf{u}_{m}(t)-\mathbf{u}(t)+\varepsilon \mathbf{w}(t)\right) \mathrm{d} t \geq 0 .
$$

Passing to the limit, we derive

$$
\int_{0}^{T}(\boldsymbol{\Psi}(t)-\mathbf{K}(\mathbf{u}(t)), \mathbf{w}(t)) \mathrm{d} t=0
$$

and, finally

$$
\lim _{m \rightarrow \infty} \mathbf{K}\left(\mathbf{u}_{m}\right)=\mathbf{K}(\mathbf{u}) \text { weak in } L^{2}\left(0, T ; L^{2}(\Omega)^{3}\right) .
$$

From there we readily pass to the limit in (4.4) and derive that $\mathbf{u}$ is the solution of problem (2.20, 2.4). Since this solution is unique, the whole sequence $\left\{\mathbf{u}_{m}\right\}$ tends to $\mathbf{u}$. This establishes the main theorem of this section. 
Theorem 4.7. Under the assumptions of Theorem 4.6, the problem (2.20, 2.4) has one and only one solution $\mathbf{u}$ that exists for all time $t \geq 0$. Furthermore, $\mathbf{u}$ belongs to $L^{\infty}\left(\mathbb{R}^{+} ; V_{2}\right)$, $\mathbf{u}^{\prime}$ belongs to $L^{2}\left(\mathbb{R}^{+} ; V\right)$ and $\mathbf{u}$ is uniformly bounded in $V_{2}$ with respect to time:

$$
\|\mathbf{u}\|_{L^{\infty}\left(\mathbb{R}^{+} ; V_{2}\right)} \leq \min \left(\sqrt{\frac{\nu}{2 \alpha_{1} C\left(\alpha_{1}, \alpha_{2}, \beta\right)}}, \frac{K_{1}}{K_{2}}\right)
$$

where $C\left(\alpha_{1}, \alpha_{2}, \beta\right)$ is defined by (3.10) and $K_{1}$ and $K_{2}$ are defined as in Lemma 3.1.

\section{Additional Regularity: The Classical SOLUtion}

In this section, we assume that problem $(2.20,2.4)$ has a solution $\mathbf{u}$ in $L^{\infty}\left(0, T ; V_{2}\right)$ with $\mathbf{u}^{\prime}$ in $L^{2}(0, T ; V)$, which is not necessarily global. Let us take both $\mathbf{f}$ and curl $\mathbf{f}$ in $L^{1}\left(0, T ; H^{1}(\Omega)^{3}\right)$, $\mathbf{u}_{0}$ in $H^{4}(\Omega)^{3} \cap V$ and $\Gamma$ of class $C^{3,1}$. We propose to show that $\operatorname{curl}\left(\mathbf{u}-\alpha_{1} \Delta \mathbf{u}\right)$ belongs to $L^{\infty}\left(0, T ; H^{1}(\Omega)^{3}\right)$. In view of Remark 2.3 , this implies that $\mathbf{u}$ is in $L^{\infty}\left(0, T ; H^{4}(\Omega)^{3}\right)$.

First, we are going to define two linear mappings $\mathbf{l}$ and $\mathbf{g}$ defined in $L^{2}(\Omega)^{3}$ such that $\mathbf{l}\left(\operatorname{curl}\left(\mathbf{u}-\alpha_{1} \Delta \mathbf{u}\right)\right)=\mathbf{u}$ and $\mathbf{g}\left(\operatorname{curl}\left(\mathbf{u}-\alpha_{1} \Delta \mathbf{u}\right)\right)=\mathbf{c u r l} \mathbf{u}$. Afterwards, from equation (2.30) and using $\mathbf{l}$ and $\mathbf{g}$, we shall derive a transport equation with particular solution $\mathbf{z}=\operatorname{curl}\left(\mathbf{u}-\alpha_{1} \Delta \mathbf{u}\right)$ in $L^{2}\left(0, T ; L^{2}(\Omega)^{3}\right)$. Then we shall show that this equation has a solution in $L^{\infty}\left(0, T ; H^{1}(\Omega)^{3}\right)$ and finally that it has no more than one solution in $L^{2}\left(0, T ; L^{2}(\Omega)^{3}\right)$. Therefore the unique solution $\mathbf{z}$ in $L^{\infty}\left(0, T ; H^{1}(\Omega)^{3}\right)$ is $\operatorname{curl}\left(\mathbf{u}-\alpha_{1} \Delta \mathbf{u}\right)$, hence $\mathbf{u}$ belongs to $L^{\infty}\left(0, T ; H^{4}(\Omega)^{3}\right)$.

\subsection{A transport equation}

We define

$$
G=\left\{\mathbf{v} \in L^{2}(\Omega)^{3} ; \operatorname{div} \mathbf{v}=0,<\mathbf{v} \cdot \mathbf{n}, 1>_{\Gamma_{i}}=0 \text { for } 0 \leq i \leq p\right\} .
$$

Let $P_{G}$ be the orthogonal projection operator on $\mathrm{G}$ for the scalar product in $L^{2}(\Omega)^{3}$. Let $\mathbf{z}$ belong to $L^{2}(\Omega)^{3}$ and set

$$
\mathbf{y}_{\mathbf{z}}=P_{G}(\mathbf{z})
$$

As $\Omega$ is simply-connected, there exists a unique vector-potential $\phi_{\mathbf{z}}$ such that

$$
\mathbf{y}_{\mathbf{z}}=\operatorname{curl} \phi_{\mathbf{z}} \text { and } \operatorname{div} \phi_{\mathbf{z}}=0 \text { in } \Omega, \phi_{\mathbf{z}} \cdot \mathbf{n}=0 \text { on } \Gamma \text {. }
$$

Furthermore, the regularity of $\Gamma$ implies that $\phi_{\mathbf{z}}$ belongs to $H^{1}(\Omega)^{3}$ and there exists a constant $C_{1}^{\prime}$ such that

$$
\left\|\phi_{\mathbf{z}}\right\|_{H^{1}(\Omega)} \leq C_{1}^{\prime}\left\|\mathbf{y}_{\mathbf{z}}\right\|_{L^{2}(\Omega)} \leq C_{1}^{\prime}\|\mathbf{z}\|_{L^{2}(\Omega)} .
$$

Then we define $\mathbf{v}_{\mathbf{z}}$ in $\mathrm{V}$ as the solution of the Stokes problem

$$
\mathbf{v}_{\mathbf{z}}-\alpha_{1} \Delta \mathbf{v}_{\mathbf{z}}+\nabla \pi_{\mathbf{z}}=\phi_{\mathbf{z}}
$$

The regularity of $\Gamma$ implies that $\mathbf{v}_{\mathbf{z}} \in H^{3}(\Omega)^{3}$ and there exists a constant $C_{2}^{\prime}$ such that

$$
\left\|\mathbf{v}_{\mathbf{z}}\right\|_{H^{3}(\Omega)} \leq C_{2}^{\prime}\left\|\phi_{\mathbf{z}}\right\|_{H^{1}(\Omega)} .
$$

We set

$$
\mathbf{l}(\mathbf{z})=\mathbf{v}_{\mathbf{z}} \text { and } \mathbf{g}(\mathbf{z})=\operatorname{curl} \mathbf{v}_{\mathbf{z}}
$$


Then the above inequalities enable us to obtain

$$
\|\mathbf{l}(\mathbf{z})\|_{H^{3}(\Omega)} \leq C^{\prime}\|\mathbf{z}\|_{L^{2}(\Omega)} \text { and }\|\mathbf{g}(\mathbf{z})\|_{H^{2}(\Omega)} \leq \sqrt{2} C^{\prime}\|\mathbf{z}\|_{L^{2}(\Omega)} .
$$

Lemma 5.1. Let $\mathbf{l}$ and $\mathbf{g}$ be defined by (5.1). If $\mathbf{u} \in V_{2}$, then

$$
\mathbf{l}\left(\operatorname{curl}\left(\mathbf{u}-\alpha_{1} \Delta \mathbf{u}\right)\right)=\mathbf{u} \text { and } \mathbf{g}\left(\operatorname{curl}\left(\mathbf{u}-\alpha_{1} \Delta \mathbf{u}\right)\right)=\operatorname{curl} \mathbf{u} .
$$

Proof. If $\mathbf{z}=\operatorname{curl}\left(\mathbf{u}-\alpha_{1} \Delta \mathbf{u}\right)$, then $\operatorname{curl}\left(\mathbf{u}-\alpha_{1} \Delta \mathbf{u}\right)=\operatorname{curl}\left(\mathbf{v}_{\mathbf{z}}-\alpha_{1} \Delta \mathbf{v}_{\mathbf{z}}\right)$. Hence $\mathbf{v}_{\mathbf{z}}=\mathbf{u}$.

Lemma 5.2. In addition to the assumptions of Lemma 2.1, suppose that $\Gamma$ is of class $C^{3,1}$. Then the mapping $\mathbf{l}$ is a linear continuous operator from $H^{1}(\Omega)^{3}$ into $H^{4}(\Omega)^{3}$ and the mapping $\mathbf{g}$ is a linear continuous operator from $H^{1}(\Omega)^{3}$ into $H^{3}(\Omega)^{3}$ and there exists a constant $C^{\prime \prime}$ such that

$$
\|\mathbf{l}(\mathbf{z})\|_{H^{4}(\Omega)} \leq C^{\prime \prime}\|\mathbf{z}\|_{H^{1}(\Omega)} \text { and }\|\mathbf{g}(\mathbf{z})\|_{H^{3}(\Omega)} \leq \sqrt{2} C^{\prime \prime}\|\mathbf{z}\|_{H^{1}(\Omega)} .
$$

Proof. Let $\mathbf{z}$ belong to $H^{1}(\Omega)^{3}$

$$
\mathbf{z}=\mathbf{y}_{\mathbf{z}}+\mathbf{w}_{\mathbf{z}}
$$

where $\mathbf{y}_{\mathbf{z}} \in G$ and $\mathbf{w}_{\mathbf{z}} \in G^{\perp}$. It can be proved that

$$
\mathbf{w}_{\mathbf{z}}=\nabla p_{\mathbf{z}}+\sum_{i=1}^{p}<\left(\mathbf{z}-\nabla p_{\mathbf{z}}\right) \cdot \mathbf{n}, 1>_{\Gamma_{i}} \nabla q_{i}^{N}
$$

where $p_{\mathbf{z}}$ is the solution of the Dirichlet problem:

$$
\Delta p_{\mathbf{z}}=\operatorname{div} \mathbf{z} \quad \text { with } p_{\mathbf{z}} \in H_{0}^{1}(\Omega)
$$

and each $q_{i}^{N}$ is the unique solution in $H^{1}(\Omega)$ of the problem (cf. [2])

$$
\left\{\begin{array}{l}
-\Delta q_{i}^{N}=0 \text { in } \Omega \\
\left.q_{i}^{N}\right|_{\Gamma_{0}}=0 \text { and }\left.q_{i}^{N}\right|_{\Gamma_{k}}=\text { constant, } 1 \leq k \leq p, \\
\left\langle\nabla q_{i}^{N} . \mathbf{n}, 1\right\rangle_{\Gamma_{0}}=-1 \text { and }\left\langle\nabla q_{i}^{N} . \mathbf{n}, 1\right\rangle_{\Gamma_{k}}=\delta_{i k}, 1 \leq k \leq p .
\end{array}\right.
$$

Owing to the regularity of the Dirichlet problem and the fact that $\nabla q_{i}^{N} \in H^{m}(\Omega)^{3}$, provided $\Gamma$ is of class $C^{m, 1}$ for an integer $m \geq 1$, we obtain that the mapping $\mathbf{z} \longmapsto \mathbf{w}_{\mathbf{z}}$ is a linear continuous operator from $H^{1}(\Omega)^{3}$ into itself. Hence there exists a constant $C_{0}^{\prime \prime}$ such that

$$
\forall \mathbf{z} \in H^{1}(\Omega)^{3},\left\|\mathbf{y}_{\mathbf{z}}\right\|_{H^{1}(\Omega)} \leq C_{0}^{\prime \prime}\|\mathbf{z}\|_{H^{1}(\Omega)} .
$$

Then the regularity of $\Gamma$ implies that there exists a constant $C_{1}^{\prime \prime}$ such that

$$
\forall \mathbf{z} \in H^{1}(\Omega)^{3},\left\|\mathbf{v}_{\mathbf{z}}\right\|_{H^{4}(\Omega)} \leq C_{1}^{\prime \prime}\left\|\mathbf{y}_{\mathbf{z}}\right\|_{H^{1}(\Omega)} .
$$

To transform equation (2.30) into a more adequate equation, we shall replace $\boldsymbol{\omega}-\alpha_{1} \Delta \boldsymbol{\omega}$ by $\mathbf{z}$ and $\boldsymbol{\omega}$ by $\mathbf{g}(\mathbf{z})$ where $\boldsymbol{\omega}=$ curl $\mathbf{u}$. But expressing $\sum_{k=1}^{3} \nabla u_{k} \times \nabla \Delta u_{k}$ or $\mathbf{B}(\mathbf{u})$ as a bilinear function of $\mathbf{z}$ and $\mathbf{u}$ is not 
straightforward and to this end we need the following results that we can verify on remembering that $\operatorname{div} \mathbf{u}=0$.

$$
\sum_{k=1}^{3} \nabla u_{k} \times \nabla \Delta u_{k}=-\sum_{k=1}^{3} \frac{\partial \mathbf{u}}{\partial x_{k}} \times \operatorname{curl}\left(\frac{\partial \boldsymbol{\omega}}{\partial x_{k}}\right)+\Delta \boldsymbol{\omega} \cdot \nabla \mathbf{u}-\boldsymbol{\omega} \cdot \nabla \Delta \mathbf{u}+\Delta \boldsymbol{\omega} \times \boldsymbol{\omega},
$$

$$
\Delta \mathbf{u} \cdot \nabla \boldsymbol{\omega}+\boldsymbol{\omega} \cdot \nabla \Delta \mathbf{u}=-\nabla(\operatorname{curl} \boldsymbol{\omega} . \operatorname{curl} \mathbf{u})-\frac{1}{\alpha_{1}}\left(\boldsymbol{\omega}-\alpha_{1} \Delta \boldsymbol{\omega}\right) \times \operatorname{curl} \mathbf{u},
$$

$$
\Delta \boldsymbol{\omega} \cdot \nabla \mathbf{u}=-\frac{1}{\alpha_{1}}\left(\boldsymbol{\omega}-\alpha_{1} \Delta \boldsymbol{\omega}\right) . \nabla \mathbf{u}+\frac{1}{\alpha_{1}} \operatorname{curl} \mathbf{u} . \nabla \mathbf{u} .
$$

Next, we set

$$
\mathbf{L}(\mathbf{u}, \mathbf{v}, \mathbf{z})=2 \sum_{i, j, k=1}^{3}\left[\nabla\left(A_{i j}(\mathbf{u}) \frac{\partial}{\partial x_{k}}\left(A_{i j}(\mathbf{l}(\mathbf{z}))\right)\right) \times A_{., k}(\mathbf{v})-\frac{\partial}{\partial x_{k}}\left(A_{i j}(\mathbf{u})\right) A_{i j}(\mathbf{v}) \nabla\left((\mathbf{g}(\mathbf{z}))_{k}\right)\right] .
$$

From Lemma 5.1, we derive

$$
\left.\mathbf{L}\left(\mathbf{u}, \mathbf{u}, \boldsymbol{\omega}-\alpha_{1} \Delta \boldsymbol{\omega}\right)\right)=\mathbf{B}(\mathbf{u})
$$

On using $(5.4-5.6,5.8)$ in the transport equation $(2.30)$, we obtain

$$
\begin{aligned}
\frac{\partial}{\partial t}\left(\boldsymbol{\omega}-\alpha_{1} \Delta \boldsymbol{\omega}\right)+\frac{\nu}{\alpha_{1}}(\boldsymbol{\omega} & \left.-\alpha_{1} \Delta \boldsymbol{\omega}\right)+\mathbf{u} \cdot \nabla\left(\boldsymbol{\omega}-\alpha_{1} \Delta \boldsymbol{\omega}\right)-\frac{3 \alpha_{1}+2 \alpha_{2}}{\alpha_{1}}\left(\boldsymbol{\omega}-\alpha_{1} \Delta \boldsymbol{\omega}\right) . \nabla \mathbf{u} \\
& +\left(\alpha_{1}+\alpha_{2}\right) \nabla(\mathbf{c u r l} \boldsymbol{\omega} . \operatorname{curl} \mathbf{u})-\frac{\alpha_{1}+\alpha_{2}}{\alpha_{1}}\left(\boldsymbol{\omega}-\alpha_{1} \Delta \boldsymbol{\omega}\right) \times \operatorname{curl} \mathbf{u} \\
& +2\left(\alpha_{1}+\alpha_{2}\right) \sum_{k=1}^{3}\left(\frac{\partial \boldsymbol{\omega}}{\partial x_{k}} \cdot \nabla \frac{\partial \mathbf{u}}{\partial x_{k}}-\frac{\partial \mathbf{u}}{\partial x_{k}} \cdot \nabla \frac{\partial \boldsymbol{\omega}}{\partial x_{k}}-\frac{\partial \mathbf{u}}{\partial x_{k}} \times \operatorname{curl}\left(\frac{\partial \boldsymbol{\omega}}{\partial x_{k}}\right)\right) \\
+ & \beta\left(\frac{1}{\alpha_{1}}|A(\mathbf{u})|^{2}\left(\boldsymbol{\omega}-\alpha_{1} \Delta \boldsymbol{\omega}\right)-2 \nabla\left(|A(\mathbf{u})|^{2}\right) \cdot \nabla \boldsymbol{\omega}-\mathbf{L}\left(\mathbf{u}, \mathbf{u}, \boldsymbol{\omega}-\alpha_{1} \Delta \boldsymbol{\omega}\right)\right) \\
= & \operatorname{curl} \mathbf{f}+\frac{\nu}{\alpha_{1}} \operatorname{curl} \mathbf{u}-2 \frac{\alpha_{1}+\alpha_{2}}{\alpha_{1}} \operatorname{curl} \mathbf{u} \cdot \nabla \mathbf{u}+\frac{\beta}{\alpha_{1}}|A(\mathbf{u})|^{2} \mathbf{c u r l} \mathbf{u} .
\end{aligned}
$$

Since we know that the solution $\mathbf{u}$ of problem $(2.20,2.4)$ exists, the previous equation leads us to solve the following transport equation, obtained by replacing $\boldsymbol{\omega}-\alpha_{1} \Delta \boldsymbol{\omega}$ by $\mathbf{z}$ and $\boldsymbol{\omega}$ by $\mathbf{g}(\mathbf{z})$ :

For $\mathbf{u}$ given in $L^{\infty}\left(0, T ; V_{2}\right), \mathbf{u}_{0}$ given in $H^{4}(\Omega)^{3} \cap V$ and $\mathbf{f}$ given such that curl $\mathbf{f}$ belongs to $L^{1}\left(0, T ; H^{1}(\Omega)^{3}\right)$, find $\mathbf{z}$ in $L^{\infty}\left(0, T ; H^{1}(\Omega)^{3}\right)$ solution of:

$$
\begin{aligned}
\frac{\partial \mathbf{z}}{\partial t}+\frac{\nu}{\alpha_{1}} \mathbf{z}+\mathbf{u} \cdot \nabla \mathbf{z}-\frac{3 \alpha_{1}+2 \alpha_{2}}{\alpha_{1}} \mathbf{z} \cdot \nabla \mathbf{u}+\left(\alpha_{1}+\alpha_{2}\right)[\nabla(\operatorname{curl} \mathbf{g}(\mathbf{z}) \cdot \mathbf{c u r l} \mathbf{u}) \\
\left.-\frac{1}{\alpha_{1}} \mathbf{z} \times \operatorname{curl} \mathbf{u}+2 \sum_{k=1}^{3}\left(\frac{\partial}{\partial x_{k}} \mathbf{g}(\mathbf{z}) \cdot \nabla \frac{\partial \mathbf{u}}{\partial x_{k}}-\frac{\partial \mathbf{u}}{\partial x_{k}} \cdot \nabla \frac{\partial}{\partial x_{k}} \mathbf{g}(\mathbf{z})-\frac{\partial \mathbf{u}}{\partial x_{k}} \times \frac{\partial}{\partial x_{k}} \operatorname{curl} \mathbf{g}(\mathbf{z})\right)\right] \\
+\frac{\beta}{\alpha_{1}}|A(\mathbf{u})|^{2} \mathbf{z}-2 \beta \nabla\left(|A(\mathbf{u})|^{2}\right) . \nabla \mathbf{g}(\mathbf{z})-\beta \mathbf{L}(\mathbf{u}, \mathbf{u}, \mathbf{z}) \\
=\operatorname{curl} \mathbf{f}+\frac{\nu}{\alpha_{1}} \operatorname{curl} \mathbf{u}-2 \frac{\alpha_{1}+\alpha_{2}}{\alpha_{1}} \operatorname{curl} \mathbf{u} . \nabla \mathbf{u}+\frac{\beta}{\alpha_{1}}|A(\mathbf{u})|^{2} \operatorname{curl} \mathbf{u},
\end{aligned}
$$




$$
\mathbf{z}(0)=\operatorname{curl}\left(\mathbf{u}_{0}-\alpha_{1} \Delta \mathbf{u}_{0}\right) .
$$

Let us recall that, by construction, if $\mathbf{u}$ is a solution of $(2.20,2.4)$, with $\mathbf{u}^{\prime}$ in $L^{2}(0, T ; V)$, then $\mathbf{z}=\mathbf{c u r l}\left(\mathbf{u}-\alpha_{1} \Delta \mathbf{u}\right)$ is a solution in $L^{2}\left(0, T ; L^{2}(\Omega)^{3}\right)$ of $(5.9,5.10)$.

\subsection{Existence of solution in $L^{\infty}\left(0, T ; H^{1}(\Omega)^{3}\right)$ for the transport equation}

In order to construct a solution of $(5.9,5.10)$, let us discretize it by Galerkin's method with a basis similar to the one introduced by Temam in [15]. The spectral problem

$$
\forall \mathbf{v} \in H^{1}(\Omega)^{3},(\mathbf{w}, \mathbf{v})+(\nabla \mathbf{w}, \nabla \mathbf{v})=\lambda(\mathbf{w}, \mathbf{v})
$$

has a countable sequence of distinct positive eigenvalues:

$$
0<\lambda_{1}<\lambda_{2}<\ldots<\lambda_{k} \rightarrow \infty
$$

and a corresponding set of eigenfunctions $\left\{\mathbf{w}_{j}\right\}_{j \geq 1}$ that form an orthonormal basis of $L^{2}(\Omega)^{3}$ and an orthogonal basis of $H^{1}(\Omega)^{3}$. Moreover $\mathbf{w}_{j} \in H^{2}(\Omega)^{3}$, provided $\Gamma$ is of class $C^{1,1}$ (homogeneous Neumann problem).

For $m \geq 1$, we denote by $X_{m}$ the space spanned by $\left\{\mathbf{w}_{j}\right\}_{j=1}^{m}$ and by $P_{m}$ the orthogonal projection operator on $X_{m}$ for the scalar product of $H^{1}(\Omega)^{3}$. Then problem $(5.9,5.10)$ is discretized by: Find

$$
\mathbf{z}_{m}(t)=\sum_{j=1}^{m} c_{j, m}(t) \mathbf{w}_{j}
$$

solution, for $1 \leq j \leq m$, of

$$
\begin{gathered}
\left(\mathbf{z}_{m}^{\prime}(t), \mathbf{w}_{j}\right)+\frac{\nu}{\alpha_{1}}\left(\mathbf{z}_{m}(t), \mathbf{w}_{j}\right)+b\left(\mathbf{u}(t) ; \mathbf{z}_{m}(t), \mathbf{w}_{j}\right) \\
-\frac{3 \alpha_{1}+2 \alpha_{2}}{\alpha_{1}} b\left(\mathbf{z}_{m}(t) ; \mathbf{u}(t), \mathbf{w}_{j}\right)+\left(\alpha_{1}+\alpha_{2}\right)\left\{\left(\nabla\left(\operatorname{curl} \mathbf{g}\left(\mathbf{z}_{m}(t)\right) \cdot \mathbf{c u r l} \mathbf{u}(t)\right), \mathbf{w}_{j}\right)\right. \\
\quad-\frac{1}{\alpha_{1}}\left(\mathbf{z}_{m}(t) \times \operatorname{curl} \mathbf{u}(t), \mathbf{w}_{j}\right)+2 \sum_{k=1}^{3}\left[b\left(\frac{\partial}{\partial x_{k}} \mathbf{g}\left(\mathbf{z}_{m}(t)\right) ; \frac{\partial \mathbf{u}(t)}{\partial x_{k}}, \mathbf{w}_{j}\right)\right. \\
\left.\left.\quad-b\left(\frac{\partial \mathbf{u}(t)}{\partial x_{k}} ; \frac{\partial}{\partial x_{k}} \mathbf{g}\left(\mathbf{z}_{m}(t)\right), \mathbf{w}_{j}\right)-\left(\frac{\partial \mathbf{u}(t)}{\partial x_{k}} \times \frac{\partial}{\partial x_{k}} \operatorname{curl} \mathbf{g}\left(\mathbf{z}_{m}(t)\right), \mathbf{w}_{j}\right)\right]\right\} \\
+\beta\left(\frac{1}{\alpha_{1}}|A(\mathbf{u}(t))|^{2} \mathbf{z}_{m}(t)-2 \nabla\left(|A(\mathbf{u}(t))|^{2}\right) . \nabla \mathbf{g}\left(\mathbf{z}_{m}(t)\right)-\mathbf{L}\left(\mathbf{u}(t), \mathbf{u}(t), \mathbf{z}_{m}(t)\right), \mathbf{w}_{j}\right) \\
=\left(\operatorname{curl}\left(\mathbf{f}(t)+\frac{\nu}{\alpha_{1}} \mathbf{u}(t)\right)-2 \frac{\alpha_{1}+\alpha_{2}}{\alpha_{1}} \operatorname{curl} \mathbf{u}(t) . \nabla \mathbf{u}(t)+\frac{\beta}{\alpha_{1}}|A(\mathbf{u}(t))|^{2} \mathbf{c u r l} \mathbf{u}(t), \mathbf{w}_{j}\right), \\
\mathbf{z}_{m}(0)=P_{m}(\mathbf{z}(0)) .
\end{gathered}
$$

This system ( $c f .[8])$ has a solution $\mathbf{z}_{m}(t)$, unique and continuous on the whole interval $[0, T]$.

The following lemma gives bounds that we shall use frequently.

Lemma 5.3. Let the matrix $A(\mathbf{v})$ be defined by (2.18). For any $\mathbf{v}$ in $H^{3}(\Omega)^{3}$,

$$
\begin{aligned}
\|A(\mathbf{v})\|_{L^{\infty}(\Omega)}=\|A(\mathbf{v})\|_{L^{\infty}(\Omega)} & \leq 2 C_{1}\|\mathbf{v}\|_{H^{3}(\Omega)}, \\
\|\nabla A(\mathbf{v})\|_{H^{1}(\Omega)} & \leq 2\|\mathbf{v}\|_{H^{3}(\Omega)} \\
\|\nabla A(\mathbf{v})\|_{L^{4}(\Omega)} & \leq 2 C_{2}^{3 / 4}\|\mathbf{v}\|_{H^{3}(\Omega)}
\end{aligned}
$$


Proof. These bounds derive mainly from $(a+b)^{2} \leq 2\left(a^{2}+b^{2}\right)$ and from the symmetry of the matrix $A(\mathbf{v})$.

Lemma 5.4. Let $\mathbf{u}$ belong to $L^{\infty}\left(0, T ; V_{2}\right)$ and let $\mathbf{f}$ be such that $\mathbf{c u r l} \mathbf{f}$ belongs to $L^{1}\left(0, T ; H^{1}(\Omega)^{3}\right)$. Then the solution $\mathbf{z}_{m}(t)$ of $(5.12,5.13)$ is bounded as follows:

$$
\forall t \in[0, T],\left\|\mathbf{z}_{m}(t)\right\|_{H^{1}(\Omega)} \leq e^{K_{T} t}\left(\left\|\mathbf{z}_{m}(0)\right\|_{H^{1}(\Omega)}+C_{T}\right),
$$

where $K_{T}$ and $C_{T}$ are two constants that depend on $T$, but not on $m$.

Proof. Multiplying both sides of (5.12) by $\lambda_{j} c_{j, m}(t)$, applying (5.11) and summing over $j$ yields, after suppressing the variable $t$ in order to simplify the notation:

$$
\begin{gathered}
\frac{1}{2} \frac{\mathrm{d}}{\mathrm{d} t}\left\|\mathbf{z}_{m}\right\|_{H^{1}(\Omega)}^{2}+\frac{\nu}{\alpha_{1}}\left\|\mathbf{z}_{m}\right\|_{H^{1}(\Omega)}^{2}+\left(\nabla\left(\mathbf{u} \cdot \nabla \mathbf{z}_{m}\right), \nabla \mathbf{z}_{m}\right)-\frac{3 \alpha_{1}+2 \alpha_{2}}{\alpha_{1}}\left[\left(\nabla\left(\mathbf{z}_{m} \cdot \nabla \mathbf{u}\right), \nabla \mathbf{z}_{m}\right)\right. \\
\left.+\left(\mathbf{z}_{m} . \nabla \mathbf{u}, \mathbf{z}_{m}\right)\right]+\left(\alpha_{1}+\alpha_{2}\right)\left\{\left(\nabla\left(\nabla\left(\operatorname{curl} \mathbf{g}\left(\mathbf{z}_{m}\right) \cdot \mathbf{c u r l} \mathbf{u}\right)\right), \nabla \mathbf{z}_{m}\right)+\left(\nabla\left(\operatorname{curl} \mathbf{g}\left(\mathbf{z}_{m}\right) \cdot \mathbf{c u r l} \mathbf{u}\right), \mathbf{z}_{m}\right)\right. \\
\quad-\frac{1}{\alpha_{1}}\left(\nabla\left(\mathbf{z}_{m} \times \operatorname{curl} \mathbf{u}\right), \nabla \mathbf{z}_{m}\right)+2 \sum_{k=1}^{3}\left[\left(\nabla\left(\frac{\partial}{\partial x_{k}} \mathbf{g}\left(\mathbf{z}_{m}\right) \cdot \nabla \frac{\partial \mathbf{u}}{\partial x_{k}}\right), \nabla \mathbf{z}_{m}\right)\right. \\
+\left(\frac{\partial}{\partial x_{k}} \mathbf{g}\left(\mathbf{z}_{m}\right) \cdot \nabla \frac{\partial \mathbf{u}}{\partial x_{k}}, \mathbf{z}_{m}\right)-\left(\nabla\left(\frac{\partial \mathbf{u}}{\partial x_{k}} \cdot \nabla \frac{\partial}{\partial x_{k}} \mathbf{g}\left(\mathbf{z}_{m}\right)\right), \nabla \mathbf{z}_{m}\right)-\left(\frac{\partial \mathbf{u}}{\partial x_{k}} \cdot \nabla \frac{\partial}{\partial x_{k}} \mathbf{g}\left(\mathbf{z}_{m}\right), \mathbf{z}_{m}\right) \\
\left.\left.\quad-\left(\nabla\left(\frac{\partial \mathbf{u}}{\partial x_{k}} \times \frac{\partial}{\partial x_{k}} \operatorname{curl} \mathbf{g}\left(\mathbf{z}_{m}\right)\right), \nabla \mathbf{z}_{m}\right)-\left(\frac{\partial \mathbf{u}}{\partial x_{k}} \times \frac{\partial}{\partial x_{k}} \operatorname{curl} \mathbf{g}\left(\mathbf{z}_{m}\right), \mathbf{z}_{m}\right)\right]\right\} \\
+\beta\left[\frac{1}{\alpha_{1}}\left(\nabla\left(|A(\mathbf{u})|^{2} \mathbf{z}_{m}\right), \nabla \mathbf{z}_{m}\right)+\frac{1}{\alpha_{1}}\left(|A(\mathbf{u})|^{2} \mathbf{z}_{m}, \mathbf{z}_{m}\right)-2\left(\nabla\left(\nabla\left(|A(\mathbf{u})|^{2}\right) \cdot \nabla \mathbf{g}\left(\mathbf{z}_{m}\right)\right), \nabla \mathbf{z}_{m}\right)\right. \\
\left.-2\left(\nabla\left(|A(\mathbf{u})|^{2}\right) \cdot \nabla \mathbf{g}\left(\mathbf{z}_{m}\right), \mathbf{z}_{m}\right)-\left(\nabla\left(\mathbf{L}\left(\mathbf{u}, \mathbf{u}, \mathbf{z}_{m}\right)\right), \nabla \mathbf{z}_{m}\right)-\left(\mathbf{L}\left(\mathbf{u}, \mathbf{u}, \mathbf{z}_{m}\right), \mathbf{z}_{m}\right)\right] \\
=\left(\mathbf{c u r l} \mathbf{f}+\frac{\nu}{\alpha_{1}} \mathbf{c u r l} \mathbf{u}-2 \frac{\alpha_{1}+\alpha_{2}}{\alpha_{1}} \mathbf{c u r l} \mathbf{u} \cdot \nabla \mathbf{u}+\frac{\beta}{\alpha_{1}}|A(\mathbf{u})|^{2} \mathbf{c u r l} \mathbf{u}, \mathbf{z}_{m}\right)_{H^{1}(\Omega)} .
\end{gathered}
$$

The only troublesome term in (5.15) is $\left(\nabla\left(\mathbf{u}(t) . \nabla \mathbf{z}_{m}(t)\right), \mathbf{z}_{m}(t)\right)$ because it involves the second derivation of $\mathbf{z}_{m}$. On expanding this term, owing to Green's formula, we obtain

$$
\left(\nabla\left(\mathbf{u} . \nabla \mathbf{z}_{m}\right), \nabla \mathbf{z}_{m}\right)=\left(\frac{\partial u_{l}}{\partial x_{k}} \frac{\partial z_{m, i}}{\partial x_{l}}, \frac{\partial z_{m, i}}{\partial x_{k}}\right)
$$

Let us examine the other terms of grade 2 (terms not derived from $\beta \operatorname{div}\left(\left|A_{1}\right|^{2} A_{1}\right)$ ) of the left-hand side. On the one hand, we have the terms

$$
\begin{gathered}
\left|\left(\mathbf{z}_{m} \cdot \nabla \mathbf{u}, \mathbf{z}_{m}\right)\right|,\left|\left(\nabla\left(\operatorname{curl} \mathbf{g}\left(\mathbf{z}_{m}\right) \cdot \mathbf{c u r l} \mathbf{u}\right), \mathbf{z}_{m}\right)\right|, \quad\left|\sum_{k=1}^{3}\left(\frac{\partial}{\partial x_{k}} \mathbf{g}\left(\mathbf{z}_{m}\right) \cdot \nabla \frac{\partial \mathbf{u}}{\partial x_{k}}, \mathbf{z}_{m}\right)\right| \\
\left|\sum_{k=1}^{3}\left(\frac{\partial \mathbf{u}}{\partial x_{k}} \cdot \nabla \frac{\partial}{\partial x_{k}} \mathbf{g}\left(\mathbf{z}_{m}\right), \mathbf{z}_{m}\right)\right| \text { and }\left|\sum_{k=1}^{3}\left(\frac{\partial \mathbf{u}}{\partial x_{k}} \times \frac{\partial}{\partial x_{k}} \operatorname{curl} \mathbf{g}\left(\mathbf{z}_{m}\right), \mathbf{z}_{m}\right)\right|
\end{gathered}
$$


which are bounded by terms of the form: $C\|\mathbf{u}(t)\|_{H^{3}(\Omega)}\left\|\mathbf{z}_{m}(t)\right\|_{L^{2}(\Omega)}^{2}$ where $C$ depends on $C_{1}, C_{2}$ and $C^{\prime}$. On the other hand, we have the remaining terms of grade 2 which are bounded by terms of the form: $C\|\mathbf{u}(t)\|_{H^{3}(\Omega)}\left|\mathbf{z}_{m}(t)\right|_{H^{1}(\Omega)}\left\|\mathbf{z}_{m}(t)\right\|_{H^{1}(\Omega)}$ where $\mathrm{C}$ depends on $C_{1}, C_{2}$ and $C^{\prime \prime}$.

Next, there remain the specific terms of grade 3 . Let us note that

$$
\begin{aligned}
\left(|A(\mathbf{u})|^{2} \mathbf{z}_{m}, \mathbf{z}_{m}\right) & =\left\||A(\mathbf{u})| \mathbf{z}_{m}\right\|_{L^{2}(\Omega)}^{2} \geq 0 \\
\left(\nabla\left(|A(\mathbf{u})|^{2} \mathbf{z}_{m}\right), \nabla \mathbf{z}_{m}\right) & =\left\||A(\mathbf{u})| \nabla \mathbf{z}_{m}\right\|_{L^{2}(\Omega)}^{2}+2 \sum_{l, k, j=1}^{3}\left(A_{l k}(\mathbf{u}) \frac{\partial A_{l k}(\mathbf{u})}{\partial x_{j}} \mathbf{z}_{m}, \frac{\partial \mathbf{z}_{m}}{\partial x_{j}}\right) .
\end{aligned}
$$

Since $\left\||A(\mathbf{u})| \nabla \mathbf{z}_{m}\right\|_{L^{2}(\Omega)}^{2} \geq 0$, we have only to bound

$$
2 \sum_{l, k, j=1}^{3}\left(A_{l k}(\mathbf{u}) \frac{\partial A_{l k}(\mathbf{u})}{\partial x_{j}} \mathbf{z}_{m}, \frac{\partial \mathbf{z}_{m}}{\partial x_{j}}\right) .
$$

Let us consider the other terms of grade 3 of the left-hand side of (5.15). First, we have the terms

$$
\left|\left(\nabla\left(|A(\mathbf{u})|^{2}\right) . \nabla \mathbf{g}\left(\mathbf{z}_{m}\right), \mathbf{z}_{m}\right)\right|,\left|\left(\mathbf{L}\left(\mathbf{u}, \mathbf{u}, \mathbf{z}_{m}\right), \mathbf{z}_{m}\right)\right| .
$$

They are bounded by terms of the form: $C\|\mathbf{u}\|_{H^{3}(\Omega)}^{2}\left\|\mathbf{z}_{m}\right\|_{L^{2}(\Omega)}^{2}$, where $C$ depends on $C_{1}, C_{2}$ and $C^{\prime}$. There remain the terms

$$
2 \sum_{l, k, j=1}^{3}\left(A_{l k}(\mathbf{u}) \frac{\partial A_{l k}(\mathbf{u})}{\partial x_{j}} \mathbf{z}_{m}, \frac{\partial \mathbf{z}_{m}}{\partial x_{j}}\right),\left|\left(\nabla\left(\mathbf{L}\left(\mathbf{u}, \mathbf{u}, \mathbf{z}_{m}\right)\right), \nabla \mathbf{z}_{m}\right)\right|,\left|\left(\nabla\left(\nabla\left(|A(\mathbf{u})|^{2}\right) . \nabla \mathbf{g}\left(\mathbf{z}_{m}\right)\right), \nabla \mathbf{z}_{m}\right)\right| .
$$

They are bounded by terms of the form: $C\|\mathbf{u}\|_{H^{3}(\Omega)}^{2}\left\|\mathbf{z}_{m}\right\|_{H^{1}(\Omega)}\left|\mathbf{z}_{m}\right|_{H^{1}(\Omega)}$, where $C$ depends on $C_{1}, C_{2}$ and $C^{\prime \prime}$. We obtain these results by expanding the terms and by using Hölder's inequalities, Cauchy-Schwarz and the same types of arguments as in the proof of Theorem 3.3. Let us show, with an example, the arguments used on proving these types of bounds. Let us expand $\left|\left(\nabla\left(\nabla\left(|A(\mathbf{u})|^{2}\right) . \nabla \mathbf{g}\left(\mathbf{z}_{m}\right)\right), \nabla \mathbf{z}_{m}\right)\right|$.

$$
\begin{aligned}
\left(\nabla\left(\nabla\left(|A(\mathbf{u})|^{2}\right) . \nabla \mathbf{g}\left(\mathbf{z}_{m}\right)\right), \nabla \mathbf{z}_{m}\right)= & 2 \sum_{i, k, l=1}^{3}\left[b\left(\frac{\partial A_{l k}(\mathbf{u})}{\partial x_{i}} \nabla A_{l k}(\mathbf{u}) ; \mathbf{g}\left(\mathbf{z}_{m}\right), \frac{\partial \mathbf{z}_{m}}{\partial x_{i}}\right)\right. \\
& +b\left(A_{l k}(\mathbf{u}) \nabla\left(\frac{\partial A_{l k}(\mathbf{u})}{\partial x_{i}}\right) ; \mathbf{g}\left(\mathbf{z}_{m}\right), \frac{\partial \mathbf{z}_{m}}{\partial x_{i}}\right) \\
& \left.+b\left(A_{l k}(\mathbf{u}) \nabla A_{l k}(\mathbf{u}) ; \frac{\partial \mathbf{g}\left(\mathbf{z}_{m}\right)}{\partial x_{i}}, \frac{\partial \mathbf{z}_{m}}{\partial x_{i}}\right)\right]
\end{aligned}
$$

Owing to estimates with $b$ ( $c f$. the proof of Th. 3.3), Cauchy-Schwarz and (2.17), we derive

$$
\begin{aligned}
\left|\left(\nabla\left(\nabla\left(|A(\mathbf{u})|^{2}\right) . \nabla \mathbf{g}\left(\mathbf{z}_{m}\right)\right), \nabla \mathbf{z}_{m}\right)\right| \leq & 2\left|\mathbf{z}_{m}\right|_{H^{1}(\Omega)}\left[C_{2}^{3 / 2}\|\nabla A(\mathbf{u})\|_{H^{1}(\Omega)}^{2}\left\|\nabla \mathbf{g}\left(\mathbf{z}_{m}\right)\right\|_{L^{\infty}(\Omega)}\right. \\
& +\|A(\mathbf{u})\|_{L^{\infty}(\Omega)}\left(\left\|\partial^{2} A(\mathbf{u})\right\|_{L^{2}(\Omega)}\left\|\nabla \mathbf{g}\left(\mathbf{z}_{m}\right)\right\|_{L^{\infty}(\Omega)}\right. \\
& \left.\left.+C_{2}^{3 / 2}\|\nabla A(\mathbf{u})\|_{H^{1}(\Omega)}\left\|\partial^{2} \mathbf{g}\left(\mathbf{z}_{m}\right)\right\|_{H^{1}(\Omega)}\right)\right] .
\end{aligned}
$$

Then Lemma 5.3 and (5.3) give

$$
\left.\left|\left(\nabla\left(\nabla\left(|A(\mathbf{u}(t))|^{2}\right) \cdot \nabla \mathbf{g}\left(\mathbf{z}_{m}(t)\right)\right), \nabla \mathbf{z}_{m}(t)\right) \leq 8 \sqrt{2} C_{1} C^{\prime \prime}\left(2 C_{2}^{3 / 2}+C_{1}\right)\|\mathbf{u}(t)\|_{H^{3}(\Omega)}^{2}\left\|\mathbf{z}_{m}(t)\right\|_{H^{1}(\Omega)}\right| \mathbf{z}_{m}(t)\right|_{H^{1}(\Omega)} .
$$


Finally, simplifying by $\left\|\mathbf{z}_{m}(t)\right\|_{H^{1}(\Omega)}$ and using these bounds yields

$$
\begin{aligned}
\frac{\mathrm{d}}{\mathrm{d} t}\left\|\mathbf{z}_{m}(t)\right\|_{H^{1}(\Omega)} \leq & \left(K_{1}+K_{2}\|\mathbf{u}(t)\|_{H^{3}(\Omega)}\right)\|\mathbf{u}(t)\|_{H^{3}(\Omega)}\left\|\mathbf{z}_{m}(t)\right\|_{H^{1}(\Omega)}+\|\mathbf{c u r l} \mathbf{f}(t)\|_{H^{1}(\Omega)} \\
& +K_{3}\|\mathbf{u}(t)\|_{H^{3}(\Omega)}+K_{4}\|\mathbf{u}(t)\|_{H^{3}(\Omega)}^{2}+K_{5}\|\mathbf{u}(t)\|_{H^{3}(\Omega)}^{3} .
\end{aligned}
$$

Hence, (5.14) follows.

It stems from (5.14) that the sequence $\left\{\mathbf{z}_{m}\right\}$ is uniformly bounded with respect to $m$ in $L^{\infty}\left(0, T ; H^{1}(\Omega)^{3}\right)$. Hence, there exists a function $\mathbf{z}$ in $L^{\infty}\left(0, T ; H^{1}(\Omega)^{3}\right)$ and a subsequence of $\left\{\mathbf{z}_{m}\right\}$, still denoted by $\left\{\mathbf{z}_{m}\right\}$, such that

$$
\lim _{m \rightarrow \infty} \mathbf{z}_{m}=\mathbf{z} \quad \text { weakly* in } L^{\infty}\left(0, T ; H^{1}(\Omega)^{3}\right) .
$$

Because the problem is linear, we can pass easily to the limit in $(5.12,5.13)$ and show that $\mathbf{z}$ satisfies $(5.9,5.10)$. Hence, we have proved the following theorem.

Theorem 5.5. Assume that $\Omega$ is a bounded, simply-connected open set of $\mathbb{R}^{3}$ with a boundary $\Gamma$ of class $C^{3,1}$. If $\mathbf{u}$ is given in $L^{\infty}\left(0, T ; V_{2}\right), \mathbf{u}_{0}$ in $H^{4}(\Omega)^{3} \cap V$ and $\mathbf{f}$ is such that curl $\mathbf{f}$ belongs to $L^{1}\left(0, T ; H^{1}(\Omega)^{3}\right)$, then problem $(5.9,5.10)$ has at least one solution $\mathbf{z}$ in $L^{\infty}\left(0, T ; H^{1}(\Omega)^{3}\right)$.

Remark 5.6. The argument used in proving Theorem 5.5 can readily be generalized to derive a solution $\mathbf{z}$ in $L^{\infty}\left(0, T ; H^{m}(\Omega)^{3}\right)$ for any $m \geq 1$.

\subsection{Uniqueness of the solution in $L^{2}\left(0, T ; L^{2}(\Omega)^{3}\right)$ for the transport equation}

Let $\mathbf{z}_{1}$ and $\mathbf{z}_{2}$ be two solutions of $(5.9,5.10)$ and set $\boldsymbol{\zeta}=\mathbf{z}_{1}-\mathbf{z}_{2}$. Then $\boldsymbol{\zeta}$ satisfies

$$
\begin{gathered}
\frac{\partial \boldsymbol{\zeta}}{\partial t}+\frac{\nu}{\alpha_{1}} \boldsymbol{\zeta}+\mathbf{u} . \nabla \boldsymbol{\zeta}-\frac{3 \alpha_{1}+2 \alpha_{2}}{\alpha_{1}} \boldsymbol{\zeta} . \nabla \mathbf{u}+\left(\alpha_{1}+\alpha_{2}\right)\{\nabla(\operatorname{curl} \mathbf{g}(\boldsymbol{\zeta}) \cdot \operatorname{curl} \mathbf{u}) \\
\left.-\frac{1}{\alpha_{1}}(\boldsymbol{\zeta} \times \mathbf{c u r l} \mathbf{u})+2 \sum_{k=1}^{3}\left[\frac{\partial \mathbf{g}(\boldsymbol{\zeta})}{\partial x_{k}} \cdot \nabla \frac{\partial \mathbf{u}}{\partial x_{k}}-\frac{\partial \mathbf{u}}{\partial x_{k}} \cdot \nabla \frac{\partial \mathbf{g}(\boldsymbol{\zeta})}{\partial x_{k}}-\frac{\partial \mathbf{u}}{\partial x_{k}} \times \frac{\partial \operatorname{curl} \mathbf{g}(\boldsymbol{\zeta})}{\partial x_{k}}\right]\right\} \\
+\beta\left(\frac{1}{\alpha_{1}}|A(\mathbf{u})|^{2} \boldsymbol{\zeta}-2 \nabla\left(|A(\mathbf{u})|^{2}\right) . \nabla \mathbf{g}(\boldsymbol{\zeta})-\mathbf{L}(\mathbf{u}, \mathbf{u}, \boldsymbol{\zeta})\right)=0 \\
\boldsymbol{\zeta}(0)=\mathbf{0} .
\end{gathered}
$$

Because the scalar product of (5.18) with $\zeta$ is not defined, let us proceed by transposition ( $c f$. [5]). Recall that if $\boldsymbol{\zeta}$ belongs to $H^{1}(\Omega)^{3}$, owing that $\mathbf{u}$ belongs to $\mathrm{V}$, we have

$$
\forall \mathbf{v} \in H^{1}(\Omega)^{3}, \int_{\Omega}(\mathbf{u} . \nabla \boldsymbol{\zeta}) \cdot \mathbf{v} \mathrm{d} \mathbf{x}=-\int_{\Omega}(\mathbf{u} . \nabla \mathbf{v}) \cdot \boldsymbol{\zeta} \mathrm{d} \mathbf{x} .
$$

Hence problem $(5.18,5.19)$ has the equivalent variational formulation: Find $\boldsymbol{\zeta}$ in $L^{2}\left(0, T ; L^{2}(\Omega)^{3}\right)$ solution of

$$
\begin{gathered}
\forall \boldsymbol{\phi} \in L^{2}\left(0, T ; H^{1}(\Omega)^{3}\right) \text { with } \boldsymbol{\phi}^{\prime} \in L^{2}\left(0, T ; L^{2}(\Omega)^{3}\right) \text { and } \boldsymbol{\phi}(T)=\mathbf{0} \\
\int_{0}^{T}\left[\left(\boldsymbol{\zeta}(t), \boldsymbol{\phi}^{\prime}(t)-\frac{\nu}{\alpha_{1}} \boldsymbol{\phi}(t)+\mathbf{u}(t) . \nabla \boldsymbol{\phi}(t)\right)+(\mathbf{h}(\mathbf{u}, \boldsymbol{\zeta})(t), \boldsymbol{\phi}(t))\right] \mathrm{d} t=0,
\end{gathered}
$$


where

$$
\begin{aligned}
\mathbf{h}(\mathbf{u}, \boldsymbol{\zeta})= & \frac{3 \alpha_{1}+2 \alpha_{2}}{\alpha_{1}} \boldsymbol{\zeta} . \nabla \mathbf{u}-\left(\alpha_{1}+\alpha_{2}\right)\left\{\nabla(\operatorname{curl} \mathbf{g}(\boldsymbol{\zeta}) \cdot \mathbf{c u r l} \mathbf{u})-\frac{1}{\alpha_{1}}(\boldsymbol{\zeta} \times \operatorname{curl} \mathbf{u})\right. \\
& \left.+2 \sum_{k=1}^{3}\left[\frac{\partial \mathbf{g}(\boldsymbol{\zeta})}{\partial x_{k}} \cdot \nabla \frac{\partial \mathbf{u}}{\partial x_{k}}-\frac{\partial \mathbf{u}}{\partial x_{k}} \cdot \nabla \frac{\partial \mathbf{g}(\boldsymbol{\zeta})}{\partial x_{k}}-\frac{\partial \mathbf{u}}{\partial x_{k}} \times \frac{\partial \operatorname{curl} \mathbf{g}(\boldsymbol{\zeta})}{\partial x_{k}}\right]\right\} \\
& -\beta\left(\frac{1}{\alpha_{1}}|A(\mathbf{u})|^{2} \boldsymbol{\zeta}-2 \nabla\left(|A(\mathbf{u})|^{2}\right) \cdot \nabla \mathbf{g}(\boldsymbol{\zeta})-\mathbf{L}(\mathbf{u}, \mathbf{u}, \boldsymbol{\zeta})\right)
\end{aligned}
$$

with no term at $t=0$ and $t=T$ because $\boldsymbol{\zeta}(0)=\mathbf{0}$ and $\boldsymbol{\phi}(T)=\mathbf{0}$. An easy variant of Theorem 5.5 can be applied to prove that for any $\boldsymbol{\mu}$ in $L^{2}\left(0, T ; H^{1}(\Omega)^{3}\right)$, there exists a unique $\boldsymbol{\phi}$ in $L^{2}\left(0, T ; H^{1}(\Omega)^{3}\right)$ with $\phi^{\prime}$ in $L^{2}\left(0, T ; L^{2}(\Omega)^{3}\right)$ and $\phi(T)=\mathbf{0}$ such that

$$
\phi^{\prime}(t)-\frac{\nu}{\alpha_{1}} \phi(t)+\mathbf{u}(t) . \nabla \phi(t)=\boldsymbol{\mu}(t) .
$$

We set

$$
\phi=\mathbf{F}(\boldsymbol{\mu})
$$

Taking the scalar product of $(5.22)$ by $\phi(t)$ gives

$$
\forall \boldsymbol{\mu} \in L^{2}\left(0, T ; H^{1}(\Omega)^{3}\right),\|\mathbf{F}(\boldsymbol{\mu})\|_{L^{2}\left(0, T ; L^{2}(\Omega)^{3}\right)} \leq 2 T\|\boldsymbol{\mu}\|_{L^{2}\left(0, T ; L^{2}(\Omega)^{3}\right)} .
$$

Lemma 5.7. Let $\boldsymbol{\zeta}$ belong to $L^{2}\left(0, T ; L^{2}(\Omega)^{3}\right)$, $\mathbf{u}$ be given in $L^{\infty}\left(0, T ; V_{2}\right)$ and $\mathbf{h}$ be defined by (5.21). Then $\mathbf{h}(\mathbf{u}, \boldsymbol{\zeta})$ belongs to $L^{2}\left(0, T ; L^{2}(\Omega)^{3}\right)$ and

$$
\|\mathbf{h}(\mathbf{u}, \boldsymbol{\zeta})\|_{L^{2}\left(0, T ; L^{2}(\Omega)^{3}\right)} \leq C\left(\alpha_{1}\right)\|\mathbf{u}\|_{L^{\infty}\left(0, T ; V_{2}\right)}\left(D^{\prime}+D^{\prime \prime} C\left(\alpha_{1}\right)\|\mathbf{u}\|_{L^{\infty}\left(0, T ; V_{2}\right)}\right)\|\boldsymbol{\zeta}\|_{L^{2}\left(0, T ; L^{2}(\Omega)^{3}\right)},
$$

where the constants $D^{\prime}$ and $D^{\prime \prime}$ depend on $\alpha_{1}, \alpha_{2}, C_{1}, C_{2}$ and $C^{\prime}$ but not on $T$.

Proof. We use the same argument as in the preceding subsection.

The next lemma proves uniqueness in $L^{2}\left(0, T ; L^{2}(\Omega)^{3}\right)$ of the solution of problem $(5.9,5.10)$.

Lemma 5.8. Let $\Gamma$ be of class $C^{2,1}$ and let $\mathbf{u}$ be given in $L^{\infty}\left(0, T ; V_{2}\right)$. Then the only solution $\boldsymbol{\zeta}$ of $(5.18,5.19)$ in $L^{2}\left(0, T ; L^{2}(\Omega)^{3}\right)$ is $\boldsymbol{\zeta}=\mathbf{0}$.

Proof. By density, there exists a sequence $\left\{\boldsymbol{\mu}_{n}\right\}$ with $\boldsymbol{\mu}_{n} \in \mathcal{D}(] 0, T[\times \Omega)^{3}$ such that

$$
\lim _{n \rightarrow \infty}\left\|\boldsymbol{\mu}_{n}-\boldsymbol{\zeta}\right\|_{L^{2}\left(0, T ; L^{2}(\Omega)^{3}\right)}=0
$$

We set

$$
\phi_{n}=\mathbf{F}\left(\boldsymbol{\mu}_{n}\right)
$$

where $\mathbf{F}$ is defined by (5.23). On taking $\phi=\phi_{n}$ in (5.20), we obtain

$$
\int_{0}^{T}\left[\left(\boldsymbol{\zeta}(t), \boldsymbol{\phi}_{n}^{\prime}(t)-\frac{\nu}{\alpha_{1}} \boldsymbol{\phi}_{n}(t)+\mathbf{u}(t) . \nabla \boldsymbol{\phi}_{n}(t)\right)+\left(\mathbf{h}(\mathbf{u}, \boldsymbol{\zeta})(t), \boldsymbol{\phi}_{n}(t)\right)\right] \mathrm{d} t=0 .
$$


With $(5.22,5.27)$, this becomes

$$
\int_{0}^{T}\left[\left(\boldsymbol{\zeta}(t), \boldsymbol{\mu}_{n}(t)\right)+\left(\mathbf{h}(\mathbf{u}, \boldsymbol{\zeta})(t), \mathbf{F}\left(\boldsymbol{\mu}_{n}\right)(t)\right)\right] \mathrm{d} t=0 .
$$

In view of $(5.26,5.24)$, there exists a function $\psi \in L^{2}\left(0, T ; L^{2}(\Omega)^{3}\right)$ such that

$$
\lim _{n \rightarrow \infty} \mathbf{F}\left(\boldsymbol{\mu}_{n}\right)=\boldsymbol{\psi} \quad \text { weakly in } L^{2}\left(0, T ; L^{2}(\Omega)^{3}\right) .
$$

Moreover

$$
\|\boldsymbol{\psi}\|_{L^{2}\left(0, T ; L^{2}(\Omega)^{3}\right)} \leq \lim \inf \left\|\mathbf{F}\left(\boldsymbol{\mu}_{n}\right)\right\|_{L^{2}\left(0, T ; L^{2}(\Omega)^{3}\right)}
$$

Hence

$$
\|\boldsymbol{\psi}\|_{L^{2}\left(0, T ; L^{2}(\Omega)^{3}\right)} \leq 2 T\|\boldsymbol{\zeta}\|_{L^{2}\left(0, T ; L^{2}(\Omega)^{3}\right)} .
$$

Passing to the limit in $(5.28)$ gives

$$
\int_{0}^{T}\left[\|\boldsymbol{\zeta}(t)\|_{L^{2}(\Omega)}^{2}+(\mathbf{h}(\mathbf{u}, \boldsymbol{\zeta})(t), \boldsymbol{\psi}(t))\right] \mathrm{d} t=0 .
$$

Then from (5.29) and Lemma 5.7, we derive that

$$
\left.\|\boldsymbol{\zeta}\|_{L^{2}\left(0, T ; L^{2}(\Omega)^{3}\right)}^{2}\left(1-2 T C\left(\alpha_{1}\right)\|\mathbf{u}\|_{L^{\infty}\left(0, T ; V_{2}\right)}\right)\left(D^{\prime}+D^{\prime \prime} C\left(\alpha_{1}\right)\|\mathbf{u}\|_{L^{\infty}\left(0, T ; V_{2}\right)}\right)\right) \leq 0
$$

We set

$$
T^{*}=\frac{1}{4 C\left(\alpha_{1}\right)\|\mathbf{u}\|_{L^{\infty}\left(0, T ; V_{2}\right)}\left(D^{\prime}+D^{\prime \prime} C\left(\alpha_{1}\right)\|\mathbf{u}\|_{L^{\infty}\left(0, T ; V_{2}\right)}\right)} .
$$

If $T^{*} \geq T,(5.30)$ gives

$$
\boldsymbol{\zeta}(t)=\mathbf{0} \text { for all } t \text { in }[0, T]
$$

If $T^{*}<T$, there exists $p \in \mathbb{N}^{*}$ such that $(p-1) T^{*}<T \leq p T^{*}$. Replacing successively $[0, T]$ by $\left[(k-1) T^{*}, k T^{*}\right]$ for $k=1, \ldots, p-1$ in $(5.20,5.22,5.24)$ and Lemma 5.7 yields successively for $k=1, \ldots, p-1$ analogous relations to (5.30). As $\|\mathbf{u}\|_{L^{\infty}\left((k-1) T^{*}, k T^{*} ; V_{2}\right)} \leq\|\mathbf{u}\|_{L^{\infty}\left(0, T ; V_{2}\right)}$, we have

$$
\left[1-2 T^{*} C\left(\alpha_{1}\right)\|\mathbf{u}\|_{L^{\infty}\left((k-1) T^{*}, k T^{*} ; V_{2}\right)}\left(D^{\prime}+D^{\prime \prime} C\left(\alpha_{1}\right)\|\mathbf{u}\|_{L^{\infty}\left((k-1) T^{*}, k T^{*} ; V_{2}\right)}\right)\right] \geq \frac{1}{2}
$$

Hence $\boldsymbol{\zeta}(t)=\mathbf{0}$ for all $t$ in $\left[(k-1) T^{*}, k T^{*}\right]$ for $k=1, \ldots, p-1$, that is

$$
\boldsymbol{\zeta}(t)=\mathbf{0} \text { for all } t \text { in }\left[0,(p-1) T^{*}\right]
$$

Finally, the same argument on the remaining interval $\left[(p-1) T^{*}, T\right]$ gives

$$
\boldsymbol{\zeta}(t)=\mathbf{0} \text { for all } t \text { in }[0, T]
$$




\subsection{Conclusion}

Problem $(5.9,5.10)$ has a solution $\mathbf{z}$ in $L^{\infty}\left(0, T ; H^{1}(\Omega)^{3}\right)$ (see Sect. 5.2) and a solution $\mathbf{c u r l}\left(\mathbf{u}-\alpha_{1} \Delta \mathbf{u}\right)$ in $L^{2}\left(0, T ; L^{2}(\Omega)^{3}\right)$ (see Sect. 5.1). In Section 5.3, we prove that this problem has no more than one solution in $L^{2}\left(0, T ; L^{2}(\Omega)^{3}\right)$. Therefore, $\mathbf{z}=\mathbf{c u r l}\left(\mathbf{u}-\alpha_{1} \Delta \mathbf{u}\right)$ belongs to $L^{\infty}\left(0, T ; H^{1}(\Omega)^{3}\right)$ and, in view of Remark 2.3 , this implies that $\mathbf{u}$ is in $L^{\infty}\left(0, T ; H^{4}(\Omega)^{3}\right)$. We have proved the following theorem.

Theorem 5.9. Assume that $\Omega$ is a bounded, simply-connected open set of $\mathbb{R}^{3}$ with a boundary $\Gamma$. Suppose that the solution $\mathbf{u}$ of problem (2.1-2.5) belongs to $L^{\infty}\left(0, T ; V_{2}\right)$. If $\Gamma$ is of class $C^{3,1}$ and if the data have the regularity:

$$
\mathbf{u}_{0} \in H^{4}(\Omega)^{3} \cap V, \mathbf{f} \in L^{1}\left(0, T ; H^{1}(\Omega)^{3}\right), \text { curl } \mathbf{f} \in L^{1}\left(0, T ; H^{1}(\Omega)^{3}\right)
$$

then $\mathbf{u}$ belongs to $L^{\infty}\left(0, T ; H^{4}(\Omega)^{3}\right)$.

According to Remark 5.6, the statement of Theorem 5.9 can be generalized by induction to any $m \geq 1$ so that it gives the following result. Let $\Gamma$ be of class $C^{m+2,1}$ and suppose that the solution $\mathbf{u}$ of problem (2.1-2.5) belongs to $L^{\infty}\left(0, T ; V_{2}\right)$. If $\mathbf{u}_{0}$ is given in $H^{m+3}(\Omega)^{3} \cap V$ and $\mathbf{f}$ in $L^{1}\left(0, T ; H^{m}(\Omega)^{3}\right)$ with curl f in $L^{1}\left(0, T ; H^{m}(\Omega)^{3}\right)$, then $\mathbf{u}$ belongs to $L^{\infty}\left(0, T ; H^{m+3}(\Omega)^{3}\right)$. The proof is exactly the same provided we take for basis the eigenfunctions of the problem

$$
\forall \mathbf{v} \in H^{m}(\Omega)^{3},\left(\left(\mathbf{w}_{j}, \mathbf{v}\right)\right)_{m}=\lambda_{j}\left(\mathbf{w}_{j}, \mathbf{v}\right),
$$

where $((., .))_{m}$ denotes the scalar product of $H^{m}(\Omega)^{3}$.

If we apply this result for $m=4$, with $\mathbf{f}$ and curl $\mathbf{f}$ in $L^{\infty}\left(0, T ; H^{4}(\Omega)^{3}\right)$ and $\frac{\partial \mathbf{f}}{\partial t}$ in $L^{\infty}\left(0, T ; H^{3}(\Omega)^{3}\right)$, we can obtain a classical solution for problem (1.5):

$$
\mathbf{u} \in C^{1}\left([0, T] ; C^{3}(\bar{\Omega})^{3}\right), \tilde{p} \in C\left([0, T] ; C^{2}(\bar{\Omega})\right) .
$$

\section{CASE Where $\Omega$ IS NON-SIMPly-CONNECTED}

We shall only sketch the method very briefly. Let $\Omega$ be a bounded domain of $\mathbb{R}^{3}$ with $\Gamma$ at least of class $C^{1,1}$. Let $P$ be the Helmholtz's projection operator of $L^{2}(\Omega)^{3}$. Then there exist a constant $C_{1}\left(\alpha_{1}\right)$ such that

$$
\forall \mathbf{v} \in V \cap H^{2}(\Omega)^{3}, \quad\|\mathbf{v}\|_{H^{2}(\Omega)} \leq C_{1}\left(\alpha_{1}\right)\left\|P\left(\mathbf{v}-\alpha_{1} \Delta \mathbf{v}\right)\right\|_{L^{2}(\Omega)}
$$

We introduce the space

$$
V_{2}=\left\{\mathbf{v} \in V \cap H^{2}(\Omega)^{3} ; \operatorname{curl}\left(\mathbf{v}-\alpha_{1} \Delta \mathbf{v}\right) \in L^{2}(\Omega)^{3}\right\}
$$

equipped with the scalar product

$$
(\mathbf{u}, \mathbf{v})_{V_{2}}=\left(P\left(\mathbf{u}-\alpha_{1} \Delta \mathbf{u}\right), P\left(\mathbf{v}-\alpha_{1} \Delta \mathbf{v}\right)\right)+\left(\operatorname{curl}\left(\mathbf{u}-\alpha_{1} \Delta \mathbf{u}\right), \operatorname{curl}\left(\mathbf{v}-\alpha_{1} \Delta \mathbf{v}\right)\right)
$$

and associated norm $\|\mathbf{v}\|_{V_{2}}=(\mathbf{v}, \mathbf{v})_{V_{2}}^{1 / 2}$. Using the fact that the space $X_{T}(\Omega)$ is continuously imbedded in $H^{1}(\Omega)^{3}$ (cf. [2]), we can prove the following result, that is the analogue of Lemma 2.1: $V_{2} \subset H^{3}(\Omega)^{3}$ and there exist a constant $C_{2}\left(\alpha_{1}\right)$ such that

$$
\forall \mathbf{v} \in V_{2},\|\mathbf{v}\|_{H^{3}(\Omega)} \leq C_{2}\left(\alpha_{1}\right)\|\mathbf{v}\|_{V_{2}}
$$

Then, by a method analogous to that of the simply-connected case, using, in addition, some results of [2], we prove the the same types of results as in the simply-connected case about existence and uniqueness. 
Finally if we assume that the bounded domain $\Omega$, as in the geometrical examples we have in mind, satisfies the hypothesis: there exists an "admissible set of cuts" that reduces it to a simply-connected domain (see [2]), we can extend the preceding results of regularity to non-simply-connected domains.

\section{REFERENCES}

[1] C. Amrouche, Etude Globale des Fluides de Troisième Grade. Thèse de 3e cycle, Université Pierre et Marie Curie, France (1986).

[2] C. Amrouche, C. Bernardi, M. Dauge and V. Girault, Vector potentials in Three-Dimensional Nonsmooth Domains. Math. Methods Appl. Sci. 21 (1998) 823-864.

[3] C. Amrouche and D. Cioranescu, On a class of fluids of grade 3. Internat. J. Non-linear Mech. 32 (1997) 73-88.

[4] D. Bresch and J. Lemoine, On the Existence of Strong Solutions for Non-Stationary Third-Grade Fluids. Preprint, Université Blaise Pascal, Clermont-Ferrand (1996).

[5] D. Cioranescu and V. Girault, Weak and classical solutions of a family of second grade fluids. Internat. J. Non-linear Mech. 32 (1997) 317-335.

[6] D. Cioranescu and E.H. Ouazar, Existence et unicité pour les fluides de second grade. C.R. Acad. Sci. Sér. I 298 (1984) 285-287.

[7] D. Cioranescu and E.H. Ouazar, Existence and uniqueness for fluids of second grade, in Nonlinear Partial Differential Equations, Collège de France Seminar, Pitman, 109 (1984) 178-197.

[8] E.A. Coddington and N. Levinson, Theory of Ordinary Differential Equations. Mc Graw-Hill, New York (1955).

[9] R.L. Fosdick and K.R. Rajagopal, Thermodynamics and stability of fluids of third grade. Proc. Roy. Soc. London Ser. A 339 (1980) 351-377.

[10] G.P. Galdi, M. Grobbelaar-Van Dalsen and N. Sauer, Existence and uniqueness of classical solutions of the equations of motion for second grade fluids. Arch. Rational Mech. Anal. 124 (1993) 221-237.

[11] J.L. Lions, Quelques méthodes de résolution des problèmes aux limites non linéaires. Dunod, Paris (1969).

[12] J. Nečas, Les méthodes directes en théorie des équations elliptiques. Masson, Paris (1967).

[13] W. Noll and C. Truesdell, The Nonlinear Field Theory of Mechanics. Handbuch of Physik, Vol. III. Springer-Verlag, Berlin (1975).

[14] A. Sequeira and J. Videman, Global existence of classical solutions for the equations of third grade fluids. J. Math. Phys. Sci. 29 (1995) 47-69.

[15] R. Temam, Navier-Stokes Equations. North-Holland, Amsterdam (1977).

[16] J.H. Videman, Mathematical analysis of viscoelastic non-Newtonian fluids. Thesis, University of Lisbonne (1997). 